University of Nebraska - Lincoln

DigitalCommons@University of Nebraska - Lincoln

Publications from USDA-ARS / UNL Faculty

U.S. Department of Agriculture: Agricultural

Research Service, Lincoln, Nebraska

$11-18-2018$

\title{
Warm-Season Grass Monocultures and Mixtures for Sustainable Bioenergy Feedstock Production in the Midwest, USA
}

\author{
Moon-Sub Lee \\ University of Illinois at Urbana-Champaign \\ Robert B. Mitchell \\ University of Nebraska-Lincoln, rob.mitchell@ars.usda.gov \\ Emily Heaton \\ lowa State University, heaton@iastate.edu \\ Colleen Zumpf \\ University of Illinois at Urbana-Champaign \\ DoKyoung Lee \\ University of Illinois at Urbana-Champaign, leedk@illinois.edu
}

Follow this and additional works at: https://digitalcommons.unl.edu/usdaarsfacpub

Lee, Moon-Sub; Mitchell, Robert B.; Heaton, Emily; Zumpf, Colleen; and Lee, DoKyoung, "Warm-Season Grass Monocultures and Mixtures for Sustainable Bioenergy Feedstock Production in the Midwest, USA" (2018). Publications from USDA-ARS / UNL Faculty. 2162.

https://digitalcommons.unl.edu/usdaarsfacpub/2162

This Article is brought to you for free and open access by the U.S. Department of Agriculture: Agricultural Research Service, Lincoln, Nebraska at DigitalCommons@University of Nebraska - Lincoln. It has been accepted for inclusion in Publications from USDA-ARS / UNL Faculty by an authorized administrator of DigitalCommons@University of Nebraska - Lincoln. 


\title{
Warm-Season Grass Monocultures and Mixtures for Sustainable Bioenergy Feedstock Production in the Midwest, USA
}

\author{
Moon-Sub Lee ${ }^{1} \cdot$ Rob Mitchell $^{2} \cdot$ Emily Heaton $^{3} \cdot$ Colleen Zumpf $^{1} \cdot$ D. K. Lee ${ }^{1}$
}

Published online: 14 November 2018

(C) Springer Science+Business Media, LLC, part of Springer Nature 2018

\begin{abstract}
Biomass yield and adaptability to a broad range of environments are important characteristics of dedicated energy crops for sustainable bioenergy feedstock production. In addition to yield potential, the role of species diversity on ecosystem services is also growing in importance as we seek to develop sustainable feedstock production systems. The objective of this study was to compare the biomass yield potential of the commercially available germplasm of native warm-season grasses in monocultures and in blends (mixture of different cultivars of the same species) or mixtures of different species across an environmental gradient (temperature and precipitation) in the Midwest, USA. Warm-season grasses including switchgrass (Panicum virgatum L.), big bluestem (Andropogon gerardii Vitman), indiangrass (Sorghastrum nutans [L.] Nash), sideoats grama (Bouteloua curtipendula [Michx.] Torr.) and Miscanthus $\times$ giganteus (Greef and Deu.) were planted in 2009. Biomass was annually harvested from 2010 through 2015 for Urbana, IL and Mead, NE but only in 2010 and 2011 for Ames, IA. The effect of species in monocultures and mixtures (or blends) on biomass yields was significant for all locations. In monocultures, the annual biomass yields averaged over a 6-year period were $11.12 \mathrm{Mg} \mathrm{ha}^{-1}$ and $10.98 \mathrm{Mg} \mathrm{ha}^{-1}$ at Urbana and Mead, respectively, while the annual biomass yield averaged over a 2-year period was $7.99 \mathrm{Mg} \mathrm{ha}^{-1}$ at Ames, IA. Also, the annual biomass yields averaged across the different mixtures and blends at each location were $10.25 \mathrm{Mg} \mathrm{ha}^{-1}, 9.88 \mathrm{Mg} \mathrm{ha}^{-1}$, and $7.64 \mathrm{Mg} \mathrm{ha}^{-1}$ at Urbana, Mead, and Ames, respectively. At all locations, $M . \times$ giganteus and 'Kanlow N1' produced the highest biomass yield in monocultures while mixtures containing switchgrass and big bluestem had the greatest mixture yield. The results from this multi-environment study suggest mixtures of different species provided no yield advantage over monocultures for bioenergy feedstocks in Illinois and Nebraska and both systems consistently produced biomass as long as April-July precipitation was near or above the average precipitation $(300 \mathrm{~mm})$ of the regions.
\end{abstract}

Keywords Bioenergy crop $\cdot$ Warm-season grasses $\cdot$ Monoculture $\cdot$ Mixture $\cdot$ Precipitation

Moon-Sub Lee and Rob Mitchell contributed equally to this work.

Electronic supplementary material The online version of this article (https://doi.org/10.1007/s12155-018-9947-7) contains supplementary material, which is available to authorized users.

D. K. Lee

leedk@illinois.edu

1 Department of Crop Sciences, University of Illinois at Urbana-Champaign, 1120 S. Goodwin Ave, Urbana, IL 61801, USA

2 USDA/ARS Wheat, Sorghum and Forage Research Unit, 251 Filley Hall, University of Nebraska-Lincoln, East Campus,

Lincoln, NE 68583, USA

3 Department of Agronomy, Iowa State University, 716 Farm House Lane, Ames, IA, USA

\section{Introduction}

As the need for alternative, renewable energy sources increases, lignocellulosic feedstocks for second-generation bioenergy have gained attention [13, 24, 36]. Warm-season perennial grasses have been identified as potential bioenergy feedstocks due to high biomass yields, low inputs, and greater ecosystem services compared to annual crops and cool season perennial grasses [22, 24, 25]. Additionally, perennial energy feedstocks grown on marginally productive croplands could minimize competition with food production, maximize producer resources through renovation of unproductive lands, and have significant environmental benefits $[6,10,33]$.

Switchgrass (Panicum virgatum L.) and Miscanthus $\times$ giganteus (Greef and Deu.: denoted as M. $\times$ giganteus for the remainder of the paper) have received considerable interest 
as bioenergy feedstocks due to their high yield potential. Numerous studies have reported on the productivity of switchgrass and $M . \times$ giganteus, with advancements made in genetics and agronomic practices, cultivar development, and best management practices [24, 46]. However, switchgrass and $M . \times$ giganteus may not perform well across a broad environmental gradient and over multiple years $[1,7$, 34]. In contrast, relatively little research has been conducted on big bluestem (Andropogon gerardii Vitman) and indiangrass (Sorghastrum nutans [L.] Nash), although they have comparable biomass yield potentials to switchgrass $[1$, $23,34]$. The majority of information on yield potentials resulted from short-term studies and is limited to certain perennial warm-season grasses. Also, there has been relatively little research on management practices for sustainable bioenergy feedstock productions. Therefore, it is necessary to evaluate and enhance many species and cultivars for specific environments within different agro-ecoregions (Gonzalez- Hernandez et al., 2009; [1, 2]).

Environmental fluctuations such as variable temperature and precipitation patterns can have significant impacts on agricultural production $[16,34]$. The use of polycultures or multi-species swards may aid in stabilizing production across years and soil conditions $[5,16,54]$. Species diversity can enhance resilience in agricultural systems and may provide advantages for disease resistance $[19,55]$, increased production [30, 43], climate change resilience [42], and expanded ecosystem services [22].

Biomass production is the primary factor influencing selection of monocultures or mixtures for bioenergy feedstock production []. However, there are still uncertainties regarding the relationship between biomass production and species diversity. As mentioned above, Tilman et al. [43] observed that the highly diverse prairie grass mixtures produced more biomass than monocultures. Russelle et al. [35] contradicted some of the observations presented by Tilman et al. [43], specifically that difficulties in establishment and maintenance of native prairie grasses grown in high-diversity mixtures were disregarded. Schmer et al. [37] reported that switchgrass monocultures managed for high yield showed 93\% higher biomass production and equivalent net energy yield when compared to low-input high-diversity mixtures of native grassland perennials. These discrepancies may be because both studies were deficient in multi-location comparative evaluations against other high yielding perennial monocultures [21]. Therefore, the objective of this study was to compare the biomass production potential of commercially available germplasms and experimental lines of native warmseason grasses in monocultures and in blends or mixtures at three locations in the Midwest, USA, where a large portion of warm-season grasses for bioenergy feedstocks are likely to be grown. Specifically, we addressed the questions: (1) Which grass species and cultivars produce the most biomass across the sites? (2) Do monocultures produce more biomass than mixtures at each site?

\section{Materials and Methods}

The study was conducted from 2009 to 2015 in Urbana, Illinois at the University of Illinois research farm $\left(40^{\circ} 04^{\prime}\right.$ $04.0^{\prime \prime} \mathrm{N}, 88^{\circ} 11^{\prime} 43.7^{\prime \prime} \mathrm{W}$ ) on Flanagan silt loam soil (Fine, smectitic, mesic Aquic Argiudolls) and in Mead, Nebraska at the University of Nebraska research farm $\left(40^{\circ} 10^{\prime} 3.08^{\prime \prime} \mathrm{N}\right.$, $96^{\circ} 25^{\prime} 14.05^{\prime \prime} \mathrm{W}$ ) on Tomek silt loam soil (Fine, smectitic, mesic Pachic Argiudolls). The study was also conducted at a site in Ames, Iowa at the Iowa State University research farm $\left(42^{\circ} 00^{\prime} 43.9^{\prime \prime} \mathrm{N}, 93^{\circ} 44^{\prime} 33.7^{\prime \prime} \mathrm{W}\right)$, on Clarion loam soil (Fine-loamy, mixed, superactive, mesic Typic Hapludolls) but data was only collected from 2009 to 2011. All soils at each location were considered as moderate to well drained soils with a slope of less than $4 \%$ and major crops in all locations were corn and soybean until 2008. Warm-season grasses including big bluestem ('Bonanza' and 'Goldmine'), indiangrass ('Scout', 'Warrior', and 'Chief'), switchgrass ('Shawnee', 'NE2K', 'Kanlow N1', and 'Cave-in-rock'), and sideoats grama (Bouteloua curtipendula [Michx.] Torr. 'Butte') were planted as monocultures and as blends or mixtures (Table 4). Miscanthus $\times$ giganteus Greef et Deu ex. Hodkinson et Renvoize was only used as a monoculture. The mixtures were developed based on US Dept. of Agriculture Plant Hardiness Zone (PHZ) adaptations (http:// planthardiness.ars.usda.gov) and on the top yielding mixtures from previous research [44, 47-49]. 'Bonanza' big bluestem, 'Scout' indiangrass, and 'Shawnee' switchgrass were used in mixtures for PHZ 4 and 5, while 'Goldmine' big bluestem, 'Warrior' indiangrass, and 'Shawnee' switchgrass were used in mixtures for PHZ 5 and 6. 'Butte' sideoats grama was used for both PHZs. We also included a two-cultivar blend for big bluestem and indiangrass and although these two blends are technically monocultures (as they were mixtures of the same species) for the purpose of this paper, blends were analyzed as mixtures because they have wider genetic diversity than a monoculture of a single cultivar. 'Bonanza' and 'Goldmine' were used in a big bluestem blend and 'Warrior' and 'Scout' were used in an indiangrass blend. An experimental lowland switchgrass, 'NE2K', a precursor to 'Liberty' [51], was used at all sites. A total of 28 monocultures and mixtures were included in each location. Of the 18 mixtures (including the two blends) evaluated in the study, 15 of those mixtures included big bluestem and henceforth those mixtures will be denoted as the big bluestem-based mixtures.

The experimental design was a randomized complete block design with four replications at each location, and blocks were separated by alleys $(1.5 \mathrm{~m})$. The entire plot area was tilled and packed to develop a firm seedbed prior to planting. The 
individual plots $(1.5 \mathrm{~m} \times 4.5 \mathrm{~m})$ were seeded at a rate of 325 pure live seeds (PLS) $\mathrm{m}^{2}$ at a depth of $1.5 \mathrm{~cm}$ by a plot drill in Urbana, IL (Great Plain Plot planter, Salina, KS, USA), Mead, NE (Hege Inc., Waldenburg, Germany), and Ames, IA (Cole Planet JR, Cole Planter Co., GA, USA) in the spring of 2009. Also, M. $\times$ giganteus rhizomes were transplanted with $0.6 \mathrm{~m}$ row spacing and a $0.9 \mathrm{~m}$ gap between plants, at the recommended depth of 10 to $20 \mathrm{~cm}$ [31]. Quinclorac (Paramount®, (3,7-dichloro-8-quinolinecarboxylic acid)) was applied at a rate of $560 \mathrm{~g} \mathrm{ha}^{-1}$ immediately after planting for preemergent weed control. Plots were fertilized with $45 \mathrm{~kg}$ $\mathrm{P} \mathrm{ha}^{-1}$ prior to planting and with $112 \mathrm{~kg} \mathrm{~N}^{-1}$ annually beginning in the second year. In the spring of 2010, stand frequency was measured to determine grass establishment success by following the method of Vogel and Masters [45], while species composition was measured to estimate the change of species composition in monocultures and mixtures at the IL and NE sites in autumn of 2016.

From 2010 to 2015 , biomass was harvested in a $1.4 \mathrm{~m}$ wide $\times 4.0 \mathrm{~m}$ long area once annually after a killing frost. A biomass plot harvester (Cibus S, Wintersteiger, Salt Lake City, UT) was used in Urbana, IL during early December and a selfpropelled forage flail chopper (Carter Manufacturing, Brookston, IN) was employed in Mead, NE during early November at a cutting height of $10 \mathrm{~cm}$. Biomass was cut by hand in a harvest area $\left(3.72 \mathrm{~m}^{2}\right)$ per plot in Ames, IA during early December of 2010 and 2011. Fresh plot weight was measured with a combine in Urbana, IL and Mead, NE or by hand in Ames, IA where a subsample (approximately $0.5 \mathrm{~kg}$ ) was directly collected from the combine or from hand harvesting for moisture content calculation. Subsamples were dried in a forced-air oven at $55^{\circ} \mathrm{C}$ for 5 days to determine dry matter.

Weather data from stations near Urbana, IL; Mead, NE; and Ames, IA were obtained from the Illinois State Water Survey, High Plains Regional Climate Data Center, and the Iowa Environmental Mesonet, respectively. Precipitation and temperature records are shown in Tables 1 and 2 for each location for the duration of the study.

Biomass yield data were analyzed in a mixed model analysis of variance using PROC MIXED and GLIMMIX procedures in SAS (SAS institute, Cary, NC). Monoculture and mixture (including blends) treatments and year were considered as fixed effects, while block was considered to be random. Locations were analyzed separately due to interactions between location and treatments $(P<0.001)$ such as weather characteristics at each site. Contrast statements were used to compare mean biomass yields between a species and its twoand three-way mixtures using the PROC MIXED procedure in SAS. The coefficient of variance of biomass yield was calculated as the standard deviation $(\sigma)$ divided by the mean $(\mu) \times 100$. To find the critical biomass production time period relative to precipitation availability for each monoculture and mixture, a correlation coefficient was calculated across all months throughout the growing season (April to September) for each location using the CORR procedure in SAS. The monthly period with the highest correlation coefficient was deemed as the most critical time period for biomass production. All statistical significances were determined at $\alpha=0.05$.

\section{Results}

Monthly precipitation and temperature during the experimental period included a record drought in 2012 at Urbana and Mead (Tables 1 and 2). Precipitation in June and July of 2012 at Urbana was 57.9 and $15.5 \mathrm{~mm}$, respectively, which was $47 \%$ and $87 \%$ below the 30 -year average. Precipitation in July and August of 2012 at Mead was $8.4 \mathrm{~mm}$ and $7.6 \mathrm{~mm}$, which was $90 \%$ less than the 30-year average. At Ames, IA, the growing season precipitation during 2009 through 2011 did not deviate markedly from the 30 -year average, but there was approximately 5 days of flooding in 2010 at the experimental area.

The monoculture and mixtures (denoted henceforth as such) included in the study and the percentages of each species within the mixtures are outlined in Table 3 . The effect of species monoculture and mixture on biomass yield was significant in Urbana and Mead through the 6-year time period (Table 4). During the 2-year time period in Ames, the significant effect of species monoculture and mixture was also observed. The interaction between treatment $\times$ harvest year significantly affected biomass yield at Urbana and Mead, but not at Ames, while biomass yield was significantly influenced by harvest year at all locations (Table 4). However, the ratio of species composition in the mixtures did not have a significant impact on biomass yield (Table 5). The annual biomass yields in monoculture were $11.12 \mathrm{Mg} \mathrm{ha}^{-1}, 10.89 \mathrm{Mg} \mathrm{ha}^{-1}$, and $7.99 \mathrm{Mg} \mathrm{ha}^{-1}$, at Urbana, Mead, and Ames, respectively, while $10.25 \mathrm{Mg} \mathrm{ha}^{-1}, 9.88 \mathrm{Mg} \mathrm{ha}^{-1}$, and $7.64 \mathrm{Mg} \mathrm{ha}^{-1}$ were harvested in mixture at Urbana, Mead, and Ames, respectively (Table 5). M. $\times$ giganteus was the highest yielding grass grown in monoculture in all three locations across all years with an average annual yield of $17.12 \mathrm{Mg} \mathrm{ha}^{-1}$, 17.89 $\mathrm{Mg} \mathrm{ha}^{-1}$, and $13.45 \mathrm{Mg} \mathrm{ha}^{-1}$ at Urbana, Mead, and Ames, respectively. 'Kanlow N1', an experimental selection from 'Kanlow' switchgrass for improved winter survival at Mead, was the second highest yielding grass grown in monoculture with mean biomass yields of $13.86 \mathrm{Mg} \mathrm{ha}^{-1}$ at Urbana, 12.56 $\mathrm{Mg} \mathrm{ha}^{-1}$ at Mead, and $9.13 \mathrm{Mg} \mathrm{ha}^{-1}$ at Ames. Relative yields of the other treatments varied across years and locations (Table 5).

The coefficient of variation among monocultures and mixtures was different depending on location (Suppl. Table 1). Averaged across harvest years, the coefficient of variation in 
Table 1 Precipitation conditions from 2009 to 2015 with the 30-year average for Urbana, IL; Mead, NE; and Ames, IA

\begin{tabular}{|c|c|c|c|c|c|c|c|c|}
\hline Month & 2009 & 2010 & 2011 & 2012 & 2013 & 2014 & 2015 & 30 -year average \\
\hline \multicolumn{9}{|c|}{ (A) Urbana, IL } \\
\hline Jan. & 17.3 & 31.5 & 16.8 & 80.5 & 65.3 & 40.6 & 36.3 & 52.1 \\
\hline Feb. & 42.7 & 40.9 & 95.8 & 28.7 & 81.5 & 76.7 & 31.2 & 54.1 \\
\hline March & 66.5 & 73.9 & 34.5 & 41.4 & 33.8 & 35.3 & 43.2 & 72.6 \\
\hline April & 176.3 & 52.8 & 188.5 & 67.5 & 179.1 & 100.1 & 91.9 & 93.5 \\
\hline May & 145.0 & 86.6 & 125.2 & 79.0 & 95.0 & 111.3 & 154.2 & 124.2 \\
\hline June & 112.3 & 211.6 & 106.2 & 57.9 & 159.3 & 208.5 & 232.8 & 110.2 \\
\hline July & 159.8 & 95.3 & 40.1 & 15.5 & 89.7 & 221.0 & 107.2 & 119.4 \\
\hline Aug. & 142.7 & 41.6 & 44.7 & 141.2 & 9.1 & 38.6 & 80.3 & 99.8 \\
\hline Sept. & 20.3 & 81.3 & 69.3 & 145.0 & 9.7 & 87.4 & 163.6 & 79.5 \\
\hline Oct. & 223.3 & 27.9 & 62.5 & 138.7 & 91.2 & 126.0 & 31.5 & 82.8 \\
\hline Nov. & 99.6 & 98.0 & 119.9 & 27.2 & 39.1 & 61.5 & 112.0 & 93.5 \\
\hline Dec. & 95.8 & 64.8 & 69.6 & 52.6 & 56.6 & 46.0 & 189.7 & 69.3 \\
\hline \multicolumn{9}{|c|}{ (B) Mead, NE } \\
\hline Jan. & 9.7 & 20.8 & 27.2 & 4.1 & 18.5 & 6.1 & 23.1 & 16.3 \\
\hline Feb. & 16.3 & 25.1 & 20.1 & 53.3 & 13.7 & 15.7 & 23.6 & 19.6 \\
\hline March & 4.6 & 45.0 & 16.8 & 22.6 & 53.8 & 3.3 & 19.6 & 49.0 \\
\hline April & 38.6 & 64.3 & 83.1 & 88.6 & 102.1 & 88.9 & 50.5 & 68.8 \\
\hline May & 29.7 & 94.0 & 152.4 & 76.2 & 214.4 & 133.6 & 276.9 & 109.0 \\
\hline June & 157.0 & 251.5 & 87.4 & 90.7 & 63.2 & 149.9 & 194.6 & 110.2 \\
\hline July & 46.7 & 148.1 & 39.4 & 8.4 & 25.4 & 13.0 & 60.7 & 86.4 \\
\hline Aug. & 81.3 & 71.4 & 175.0 & 7.6 & 28.2 & 191.5 & 96.0 & 88.6 \\
\hline Sept. & 31.8 & 94.7 & 33.8 & 43.9 & 50.5 & 175.0 & 125.2 & 76.7 \\
\hline Oct. & 107.7 & 3.3 & 23.6 & 48.8 & 66.8 & 62.5 & 12.7 & 50.0 \\
\hline Nov. & 2.5 & 50.0 & 42.2 & 3.81 & 31.0 & 11.9 & 50.3 & 36.3 \\
\hline Dec. & 61.5 & 6.1 & 40.1 & 38.1 & 5.6 & 31.0 & 112.3 & 24.1 \\
\hline \multicolumn{9}{|c|}{ (C) Ames, IA } \\
\hline Jan. & 24.1 & 29.7 & 17.8 & & & & & 24.6 \\
\hline Feb. & 6.4 & 19.1 & 26.9 & & & & & 25.9 \\
\hline March & 103.4 & 52.6 & 20.1 & & & & & 50.3 \\
\hline April & 115.8 & 93.0 & 112.3 & & & & & 74.9 \\
\hline May & 96.0 & 92.5 & 117.3 & & & & & 104.6 \\
\hline June & 104.4 & 283.5 & 128.3 & & & & & 119.6 \\
\hline July & 69.9 & 171.2 & 99.1 & & & & & 98.8 \\
\hline Aug. & 122.9 & 284.7 & 91.2 & & & & & 96.5 \\
\hline Sept. & 24.4 & 166.9 & 51.3 & & & & & 94.0 \\
\hline Oct. & 186.2 & 9.7 & 21.8 & & & & & 59.2 \\
\hline Nov. & 35.1 & 56.6 & 69.1 & & & & & 45.0 \\
\hline Dec. & 50.0 & 20.3 & 56.6 & & & & & 27.4 \\
\hline
\end{tabular}

monoculture was $26 \%$ and $24 \%$ at Urbana and Mead, while the coefficient of variance in mixture was $28 \%$ and $20 \%$ at Urbana and Mead. There were no relationships between the coefficient of variance and species richness at any of the locations (Suppl. Fig. 2). For big bluestem-based mixtures, the two- and three-way mixture (or blends) had higher yield than the 'Bonanza' big bluestem monoculture at Urbana, while there was no yield difference between a 'Bonanza' monoculture and a mixture at Mead (Table 4). At both Urbana and Mead, the 'Goldmine' monoculture yielded the same as the mixtures (Table 4). In addition, regardless of the big bluestem and indiangrass cultivar, mixtures of big bluestem and indiangrass with 'Shawnee' switchgrass were not different from the 'Shawnee' monoculture at Urbana and Mead (Table 4). At Ames, the big bluestem two-way and three-way mixtures, similar to Urbana, had higher yields than 
Table 2 Average minimum and maximum air temperature at each location from 2009 to 2015 with the 30 -year average

Average maximum $\left({ }^{\circ} \mathrm{C}\right)$

Month
Average minimum $\left({ }^{\circ} \mathrm{C}\right)$

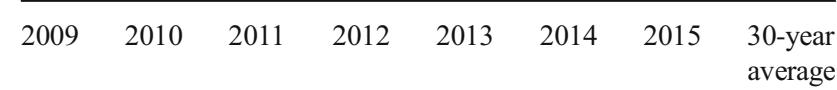

\begin{tabular}{|c|c|c|c|c|c|c|c|c|c|c|c|c|c|c|c|c|}
\hline \multirow[b]{2}{*}{ January } & \multicolumn{16}{|c|}{ Urbana, IL } \\
\hline & -4.1 & -5.1 & -3.7 & 3.3 & 1.3 & -3.6 & -1.6 & -1.2 & -13.9 & -11.7 & -11.7 & -7.2 & -8.8 & -14.2 & -10.3 & -10.2 \\
\hline February & 3.1 & -2.1 & 0.8 & 4.6 & 1.3 & -4.2 & -3.9 & 1.5 & -7.3 & -9.7 & -7.3 & -5.1 & -7.3 & -13.8 & -14.4 & -8.2 \\
\hline March & 10.9 & 10.3 & 9.0 & 17.8 & 3.8 & 5.8 & 6.9 & 8.3 & -1.6 & -0.6 & -1.7 & 4.2 & -3.0 & -6.2 & -4.2 & -2.8 \\
\hline April & 14.4 & 19.7 & 16.2 & 17.2 & 14.3 & 16.1 & 16.5 & 15.4 & 3.4 & 5.9 & 4.3 & 4.1 & 2.6 & 3.6 & 4.4 & 3.4 \\
\hline May & 21.6 & 22.1 & 20.6 & 25.4 & 22.3 & 22.2 & 22.9 & 21.3 & 9.8 & 10.8 & 9.3 & 11.7 & 10.2 & 9.9 & 11.0 & 9.2 \\
\hline June & 27.5 & 27.3 & 26.8 & 27.8 & 26.0 & 26.4 & 25.7 & 26.4 & 16.4 & 16.9 & 15.5 & 13.6 & 14.3 & 15.8 & 15.4 & 14.9 \\
\hline July & 24.6 & 28.8 & 31.4 & 33.5 & 26.1 & 24.8 & 26.6 & 27.8 & 14.3 & 17.8 & 19.4 & 18.9 & 15.6 & 13.8 & 16.1 & 16.6 \\
\hline August & 25.3 & 29.7 & 29.4 & 29.0 & 27.3 & 26.4 & 26.4 & 27.1 & 14.1 & 17.1 & 15.9 & 14.4 & 15.0 & 16.3 & 14.5 & 15.6 \\
\hline September & 23.0 & 24.7 & 22.2 & 22.7 & 26.4 & 22.7 & 25.9 & 24.0 & 12.2 & 11.3 & 10.2 & 10.4 & 12.1 & 10.1 & 12.8 & 10.7 \\
\hline October & 12.8 & 19.2 & 18.4 & 14.6 & 17.2 & 15.7 & 18.0 & 16.8 & 3.6 & 4.6 & 4.2 & 3.6 & 4.7 & 5.0 & 5.5 & 4.2 \\
\hline November & 11.4 & 10.4 & 10.8 & 8.9 & 7.2 & 4.9 & 11.7 & 8.7 & 1.0 & -2.4 & 0.8 & -2.3 & -3.6 & -5.6 & 0.2 & -1.7 \\
\hline \multirow[t]{2}{*}{ December } & 0.3 & -3.1 & 4.4 & 4.9 & -0.1 & 1.8 & 6.6 & 0.9 & -7.7 & -10.5 & -3.8 & -3.2 & -9.4 & -4.4 & -0.9 & -7.7 \\
\hline & \multicolumn{16}{|c|}{ Mead, NE } \\
\hline January & 0.9 & -5.6 & -3.7 & 4.7 & 0.5 & 0.4 & 3.3 & 0.2 & -13.3 & -13.4 & -14.7 & -10.2 & -11.4 & -14.6 & -11.0 & -11.8 \\
\hline February & 5.2 & -2.6 & 2.0 & 3.5 & 3.1 & -0.7 & -0.8 & 2.8 & -9.5 & -11.2 & -11.1 & -8.3 & -8.8 & -13.2 & -14.1 & -9.4 \\
\hline March & 10.2 & 9.2 & 8.6 & 19.2 & 6.3 & 9.5 & 14.0 & 9.6 & -4.6 & -2.6 & -3.7 & 3.0 & -6.4 & -7.1 & -4.8 & -3.9 \\
\hline April & 15.6 & 18.8 & 16.2 & 19.3 & 13.2 & 17.3 & 17.2 & 16.3 & 1.2 & 4.1 & 2.3 & 4.1 & -0.9 & 2.4 & 3.9 & 2.1 \\
\hline May & 23.4 & 20.2 & 21.4 & 25.3 & 20.9 & 24.0 & 20.1 & 21.8 & 8.7 & 8.1 & 8.3 & 10.2 & 8.4 & 9.6 & 9.3 & 8.6 \\
\hline June & 26.5 & 27.8 & 27.2 & 29.4 & 26.7 & 27.9 & 27.7 & 27.3 & 14.9 & 15.7 & 14.9 & 15.2 & 13.9 & 15.3 & 15.1 & 14.4 \\
\hline July & 27.2 & 29.0 & 31.6 & 34.4 & 29.3 & 29.3 & 29.7 & 30.0 & 14.7 & 18.6 & 20.2 & 19.0 & 16.1 & 15.2 & 17.1 & 17.3 \\
\hline August & 27.5 & 30.9 & 28.4 & 30.2 & 28.8 & 28.7 & 28.0 & 28.8 & 14.0 & 16.9 & 16.6 & 13.4 & 16.8 & 16.9 & 15.1 & 16.0 \\
\hline September & 23.2 & 24.4 & 22.3 & 26.2 & 27.0 & 23.4 & 26.4 & 24.3 & 9.4 & 10.1 & 7.2 & 6.7 & 12.6 & 10.5 & 14.1 & 10.2 \\
\hline October & 11.5 & 20.3 & 19.6 & 15.5 & 16.6 & 18.7 & 19.4 & 17.1 & 0.9 & 2.3 & 3.2 & 0.7 & 2.0 & 3.7 & 5.2 & 3.1 \\
\hline November & 12.6 & 9.0 & 9.8 & 11.8 & 8.3 & 6.4 & 11.7 & 8.5 & -2.6 & -4.4 & -4.3 & -4.7 & -6.4 & -6.9 & -0.7 & -4.1 \\
\hline \multirow[t]{2}{*}{ December } & -3.6 & 0.3 & 3.1 & 2.5 & -0.2 & 2.3 & 4.3 & 1.2 & -13.5 & -11.4 & -9.5 & -10.4 & -14.0 & -5.4 & -5.8 & -10.3 \\
\hline & \multicolumn{16}{|c|}{ Ames, IA } \\
\hline January & -6.0 & -8.2 & -6.1 & & & & & -0.6 & -16.8 & -15.3 & -15.7 & & & & & -10.0 \\
\hline February & 1.7 & -5.7 & -0.9 & & & & & 2.2 & -9.4 & -14.9 & -10.7 & & & & & -7.2 \\
\hline March & 8.0 & 6.6 & 6.1 & & & & & 9.4 & -3.7 & -2.9 & -4.2 & & & & & -1.1 \\
\hline April & 13.9 & 18.7 & 14.0 & & & & & 16.7 & 0.8 & 5.3 & 1.7 & & & & & 5.0 \\
\hline May & 20.3 & 20.7 & 20.3 & & & & & 22.2 & 8.5 & 8.7 & 7.8 & & & & & 11.1 \\
\hline June & 24.5 & 26.1 & 25.3 & & & & & 27.8 & 13.8 & 15.1 & 14.8 & & & & & 16.7 \\
\hline July & 24.4 & 27.4 & 29.3 & & & & & 30.0 & 13.4 & 17.3 & 18.8 & & & & & 19.4 \\
\hline August & 24.6 & 28.1 & 26.5 & & & & & 28.9 & 13.8 & 17.0 & 15.4 & & & & & 18.3 \\
\hline September & 22.6 & 23.2 & 21.0 & & & & & 24.4 & 10.2 & 10.4 & 8.8 & & & & & 12.8 \\
\hline October & 10.7 & 19.6 & 18.5 & & & & & 17.2 & 1.7 & 3.6 & 3.8 & & & & & 6.1 \\
\hline November & 10.6 & 7.7 & 9.1 & & & & & 8.9 & 0.1 & -3.1 & -2.1 & & & & & -0.6 \\
\hline December & -3.7 & -3.7 & 2.4 & & & & & 1.1 & -12.9 & -12.6 & -6.7 & & & & & -7.8 \\
\hline
\end{tabular}

IA, Iowa; IL, Illinois; NE, Nebraska

a 'Bonanza' monoculture. In addition, biomass yield of the big bluestem three-way mixtures were not different from the 'Shawnee' monoculture at Ames.

Biomass moisture content for the monocultures and mixtures at each location are shown in Table 6. The monocultures ranged from $17 \%$ to $32 \%$ at Urbana, whereas at Mead moisture content varied between 24 and 43\%. At both Urbana and Mead, M. $\times$ giganteus had the highest moisture content and 'Chief' indiangrass had the lowest compared to the other grasses across the years. At Ames, 
Table 3 The warm-season grass species and cultivars evaluated in the field experiment during 2009-2015 at Urbana, IL; Mead, NE; and Ames, IA

\begin{tabular}{|c|c|c|c|c|c|c|c|}
\hline \multicolumn{2}{|l|}{ Cultivar } & \multirow{2}{*}{$\begin{array}{l}\text { Abbreviation } \\
\text { MG }\end{array}$} & \multicolumn{5}{|c|}{ Species } \\
\hline \multirow[t]{11}{*}{ Monoculture } & Miscanthus $\times$ giganteus ${ }^{\ddagger}$ & & \multicolumn{5}{|c|}{ Miscanthus } \\
\hline & Cave-in-Rock & CIR & \multicolumn{5}{|c|}{ Switchgrass } \\
\hline & Kanlow N1 & KA & \multicolumn{5}{|c|}{ Switchgrass } \\
\hline & NE2K & NE2K & \multicolumn{5}{|c|}{ Switchgrass } \\
\hline & Shawnee & $\mathrm{SH}$ & \multicolumn{5}{|c|}{ Switchgrass } \\
\hline & Bonanza & $\mathrm{BO}$ & \multicolumn{5}{|c|}{ Big bluestem } \\
\hline & Goldmine & GO & \multicolumn{5}{|c|}{ Big bluestem } \\
\hline & Chief & $\mathrm{CH}$ & \multicolumn{5}{|c|}{ Indiangrass } \\
\hline & Scout & $\mathrm{SC}$ & \multicolumn{5}{|c|}{ Indiangrass } \\
\hline & Warrior & WA & \multicolumn{5}{|c|}{ Indiangrass } \\
\hline & & & \multicolumn{5}{|c|}{$\%$ in mixture ${ }^{\S}$} \\
\hline \multirow[t]{3}{*}{ Blend } & & & $\mathrm{BB}^{\dagger}$ & IN & SW & SO & MG \\
\hline & Bonanza/Goldmine & $\mathrm{BO}+\mathrm{GO}$ & 100 & 0 & 0 & 0 & 0 \\
\hline & Warrior/Scout & $\mathrm{WA}+\mathrm{SC}$ & 0 & 100 & 0 & 0 & 0 \\
\hline \multirow[t]{16}{*}{ Mixture } & Bonanza/Scout & $\mathrm{BO}+\mathrm{SC}-1$ & 40 & 60 & 0 & 0 & 0 \\
\hline & Bonanza/Scout & $\mathrm{BO}+\mathrm{SC}-2$ & 50 & 50 & 0 & 0 & 0 \\
\hline & Bonanza/Scout & $\mathrm{BO}+\mathrm{SC}-3$ & 60 & 40 & 0 & 0 & 0 \\
\hline & Bonanza/Scout/Shawnee & $\mathrm{BO}+\mathrm{SC}+\mathrm{SH}-1$ & 20 & 60 & 20 & 0 & 0 \\
\hline & Bonanza/Scout/Shawnee & $\mathrm{BO}+\mathrm{SC}+\mathrm{SH}-2$ & 40 & 40 & 20 & 0 & 0 \\
\hline & Bonanza/Scout/Shawnee & $\mathrm{BO}+\mathrm{SC}+\mathrm{SH}-3$ & 60 & 20 & 20 & 0 & 0 \\
\hline & Bonanza/Scout/Butte & $\mathrm{BO}+\mathrm{SC}+\mathrm{BU}$ & 40 & 20 & 0 & 20 & 0 \\
\hline & Goldmine/Warrior & $\mathrm{GO}+\mathrm{WA}-1$ & 40 & 60 & 0 & 0 & 0 \\
\hline & Goldmine/Warrior & $\mathrm{GO}+\mathrm{WA}-2$ & 50 & 50 & 0 & 0 & 0 \\
\hline & Goldmine/Warrior & $\mathrm{GO}+\mathrm{WA}-3$ & 60 & 40 & 0 & 0 & 0 \\
\hline & Goldmine/Warrior/Shawnee & $\mathrm{GO}+\mathrm{WA}+\mathrm{SH} 1$ & 20 & 60 & 20 & 0 & 0 \\
\hline & Goldmine/Warrior/Shawnee & $\mathrm{GO}+\mathrm{WA}+\mathrm{SH} 2$ & 40 & 40 & 20 & 0 & 0 \\
\hline & Goldmine/Warrior/Shawnee & $\mathrm{GO}+\mathrm{WA}+\mathrm{SH} 3$ & 60 & 20 & 20 & 0 & 0 \\
\hline & Goldmine/Warrior/Butte & $\mathrm{GO}+\mathrm{WA}+\mathrm{BU}$ & 40 & 20 & 0 & 20 & 0 \\
\hline & Scout/Shawnee/Butte & $\mathrm{SC}+\mathrm{SH}+\mathrm{BU}$ & 0 & 40 & 20 & 20 & 0 \\
\hline & Warrior/Shawnee/Butte & $\mathrm{WA}+\mathrm{SH}+\mathrm{BU}$ & 0 & 40 & 20 & 20 & 0 \\
\hline
\end{tabular}

${ }^{\dagger} \mathrm{BB}$, big bluestem; IN, indiangrass; SW, switchgrass; SO, sideoats grama

${ }^{\star}$ Miscanthus $\times$ giganteus clone obtained from the Chicago Botanic Garden (Glencoe, IL, USA)

$\S$ The number followed by abbreviation indicates different ratio of each species in the seeding mixture

grasses in monoculture had moisture levels ranging from 12 to $22 \%$ in 2010 and 2011.

Biomass had no consistent response to precipitation variation across the research period or across the locations. One exception was in 2012, where across many of the monocultures and mixtures, low biomass production was associated with low precipitation. Precipitation from April to July in 2012 was $50 \%$ lower than the 30-year average in Urbana and $70 \%$ lower in Mead (Fig. 1). However, at the monoculture or mixtures level, there were some feedstocks that did have significant responses to change in precipitation. At Urbana, the biomass yield for big bluestem was positively correlated with April-July precipitation ( $p$ values for 'Bonanza' 0.076 and 'Goldmine' 0.005 ), whereas no relationship was observed at Mead during the entire growing season. $M . \times$ giganteus biomass yield was positively correlated with the April-July precipitation at Mead ( $p$ value 0.014 ) though not significantly correlated at Urbana ( $p$ value 0.179 ). Switchgrass and indiangrass biomass yield were not correlated with growing season precipitation at either location.

\section{Discussion}

\section{Biomass Yield in Monocultures and Mixtures}

In previous experiments, warm-season perennial grasses have required two or more years to achieve full yield potential [17, 
Table 4 Summary of ANOVA for biomass yields of warm-season grasses and mixtures, with significant effects indicated by $P$ values, at Urbana, IL; Mead, NE; and Ames, IA. Species grown in monocultures and mixtures were considered treatments

\begin{tabular}{llll}
\hline Source of variation & Urbana, IL & Mead, NE & Ames, IW $^{*}$ \\
\hline & - & & $<.0001$ \\
Treatment $^{\dagger}$ & $<.0001$ & $<.0001$ & 0.0003 \\
Harvest year & $<.0001$ & 0.0011 & 0.1874 \\
Treatment $\times$ harvest year & $<.0001$ & 0.0004 & 0.0147 \\
Contrast & & & 0.0004 \\
BO vs. BO + SC & 0.0180 & 0.2883 & 0.8593 \\
BO vs. BO + SC + SH & $<.0001$ & 0.2472 & 0.0958 \\
SH vs. BO + SC + SH & 0.6191 & 0.8007 & 0.1682 \\
BO + SC vs. BO+SC + SH & 0.0095 & 0.8901 & 0.0020 \\
BO + SC vs. BO + SC + BU & 0.0006 & 0.3217 & 0.0021 \\
GO vs. GO + WA & 0.0790 & 0.3770 & 0.0849 \\
GO vs. GO + WA + SH & 0.1085 & 0.6661 & 0.9813 \\
SH vs. GO + WA + SH & 0.6382 & 0.8049 & 0.0225 \\
GO + WA vs. GO + WA + SH & 0.8260 & 0.5234 & 0.8197 \\
GO + WA vs. GO + WA + BU & 0.0036 & & \\
\hline
\end{tabular}

${ }^{\dagger}$ Biomass yield was averaged across species composition rates due to no differences among rates within each mixture, and analysis was tested at $\alpha=0.05$

ॠIA biomass yield data was collected in 2010 and 2011

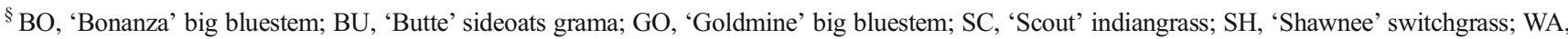
'Warrior' indiangrass

27, 29]. To maximize profits, it is important to shorten the establishment phase to achieve full yield potential as quickly as possible. In the current experiment, grass monocultures and mixtures were successfully established and reached their full production the year after planting (Suppl. Table 1). Our results indicated that warm-season grasses can be fully established in the planting year and full production could be achieved from the second year with good establishment practices including seed quality, seed bed preparation, and weed control along with favorable soil moisture conditions. In monocultures, $M . \times$ giganteus $\left(17 \mathrm{Mg} \mathrm{ha}^{-1}\right)$ and 'Kanlow N1' $\left(13 \mathrm{Mg} \mathrm{ha}^{-1}\right)$ had greater yield than other species and cultivars at Urbana, Mead, and Ames. These results agree with previous research $[8,15]$. On the other hand, Goldmine + Warrior + Shawnee $(20 / 60 / 20)$ had greater biomass yields when compared to other mixtures at Urbana and Mead and yielded more than $10 \mathrm{Mg} \mathrm{ha}^{-1}$.

\section{Biomass Moisture Content}

Biomass moisture content is critical to effective storage and transport of herbaceous feedstocks, because higher moisture content causes higher storage losses and transportation costs [39]. In our study, moisture levels were not dramatically different between monocultures and mixtures; it is noteworthy that relatively high moisture contents might result from weather conditions prior to harvesting. Recommended moisture content for storage is less than $20 \%[3,50]$, and if moisture content of biomass is above $30 \%$, harvested biomass should be windrowed to promote drying and ensure optimum moisture content for storage [50]. Danalatos et al. [4] observed an approximately $15 \%$ decrease in moisture content resulting from a two-week delayed harvest timing of $M . \times$ giganteus. Similarly, at Urbana in this study, $M . \times$ giganteus and switchgrass moisture content decreased when 2013 harvest and 2015 harvest biomass were compared, while big bluestem moisture content increased during the same experimental period. The reason for these observations could be that the 2013 biomass was harvested in November of 2013, while the 2015 biomass was collected in January of 2016. Therefore, the delayed harvest timing could lower the moisture content of $M$. $\times$ giganteus and switchgrass. However, in the case of big bluestem, lodging was caused by rain and winter snow cover before the 2015 harvest, which could have affected the moisture content in the 2015 biomass. Tahir et al. [41] also reported that early spring harvest management might be vulnerable to snow cover, lodging, and unfavorable soil conditions and therefore is a factor to take into consideration.

\section{Relationship between Biomass Yield and Seasonal Precipitation}

Previous studies have found that grassland biomass production responds positively to mean annual precipitation [28] as precipitation is one of the most important factors impacting aboveground biomass production in terrestrial ecosystems 
Table 5 Least squares means of biomass dry matter yields of warm-season grass cultivars grown in monoculture and mixtures from 2010 to 2015 at Urbana, IL and Mead, NE and from 2010 to 2011 at Ames, IA

\begin{tabular}{|c|c|c|c|c|c|}
\hline \multirow[t]{2}{*}{ Treatment } & \multirow[t]{2}{*}{$\%$ in mixture ${ }^{\S}$} & \multicolumn{4}{|c|}{ Biomass yield $\left(\mathrm{Mg} \mathrm{ha}^{-1}\right)$} \\
\hline & & Urbana, IL & Mead, NE & Ames, IA & Mean \\
\hline Miscanthus $\times$ giganteus & 100 & $17.12^{\mathrm{a} \dagger}$ & $17.89^{\mathrm{a}}$ & $13.45^{\mathrm{a}}$ & 16.15 \\
\hline Cave-In-Rock & 100 & $10.33^{\text {cde }}$ & $9.60^{\mathrm{b}}$ & $7.14^{\mathrm{bcd}}$ & 9.02 \\
\hline Kanlow N1 & 100 & $13.86^{\mathrm{b}}$ & $12.56^{\mathrm{b}}$ & $9.13^{\mathrm{b}}$ & 11.85 \\
\hline $\mathrm{NE} 2 \mathrm{~K}$ & 100 & $11.52^{\mathrm{bc}}$ & $9.94^{\mathrm{b}}$ & $6.99^{\mathrm{bcd}}$ & 9.48 \\
\hline Shawnee & 100 & $11.15^{\mathrm{cd}}$ & $10.49^{\mathrm{b}}$ & $7.43^{\mathrm{bcd}}$ & 9.69 \\
\hline Bonanza & 100 & $8.56^{\mathrm{de}}$ & $8.87^{\mathrm{b}}$ & $4.98^{\mathrm{cd}}$ & 7.54 \\
\hline Goldmine & 100 & $10.01^{\text {cde }}$ & $9.83^{\mathrm{b}}$ & $6.43^{\mathrm{bcd}}$ & 8.76 \\
\hline Chief & 100 & $8.50^{\mathrm{de}}$ & $9.14^{\mathrm{b}}$ & $7.16^{\mathrm{bcd}}$ & 8.27 \\
\hline Scout & 100 & $9.75^{\text {cde }}$ & $10.89^{\mathrm{b}}$ & $8.48^{\mathrm{b}}$ & 9.71 \\
\hline Warrior & 100 & $10.43^{\mathrm{cde}}$ & $9.76^{\mathrm{b}}$ & $8.71^{\mathrm{b}}$ & 9.63 \\
\hline Bonanza/Goldmine & $50 / 50$ & $8.96^{\text {cde }}$ & $9.02^{\mathrm{b}}$ & $4.60^{\mathrm{bcd}}$ & 7.53 \\
\hline \multirow[t]{3}{*}{ Bonanza/Scout } & $40 / 60$ & $9.99^{\text {cde }}$ & $9.47^{\mathrm{b}}$ & $6.97^{\mathrm{bcd}}$ & 8.81 \\
\hline & $50 / 50$ & $9.56^{\text {cde }}$ & $9.62^{\mathrm{b}}$ & $6.52^{\mathrm{bcd}}$ & 9.21 \\
\hline & $60 / 40$ & $10.33^{\text {cde }}$ & $9.43^{\mathrm{b}}$ & $6.67^{\mathrm{bcd}}$ & 8.71 \\
\hline \multirow[t]{3}{*}{ Bonanza/Scout/Shawnee } & $20 / 60 / 20$ & $11.10^{\mathrm{cd}}$ & $9.95^{\mathrm{b}}$ & $7.64^{\mathrm{bcd}}$ & 9.56 \\
\hline & $40 / 40 / 20$ & $10.69^{\mathrm{cd}}$ & $10.54^{\mathrm{b}}$ & $8.14^{\mathrm{bc}}$ & 9.79 \\
\hline & $60 / 20 / 20$ & $10.85^{\mathrm{cd}}$ & $10.24^{\mathrm{b}}$ & $6.87^{\mathrm{bcd}}$ & 9.32 \\
\hline Bonanza/Scout/Butte & $40 / 20 / 40$ & $7.93^{\mathrm{e}}$ & $9.18^{\mathrm{b}}$ & $5.75^{\mathrm{bcd}}$ & 7.62 \\
\hline \multirow[t]{3}{*}{ Goldmine/Warrior } & $40 / 60$ & $10.73^{\mathrm{cd}}$ & $10.21^{\mathrm{b}}$ & $9.21^{\mathrm{b}}$ & 10.77 \\
\hline & $50 / 50$ & $11.53^{\mathrm{bc}}$ & $9.83^{\mathrm{b}}$ & $8.75^{\mathrm{b}}$ & 10.04 \\
\hline & $60 / 40$ & $10.67^{\mathrm{cd}}$ & $9.88^{\mathrm{b}}$ & $8.00^{\text {bcd }}$ & 9.52 \\
\hline \multirow[t]{3}{*}{ Goldmine/Warrior/Shawnee } & $20 / 60 / 20$ & $11.28^{\mathrm{bc}}$ & $10.88^{\mathrm{b}}$ & $9.20^{\mathrm{b}}$ & 10.45 \\
\hline & $40 / 40 / 20$ & $10.90^{\mathrm{cd}}$ & $9.98^{\mathrm{b}}$ & $7.75^{\text {bcd }}$ & 9.54 \\
\hline & $60 / 20 / 20$ & $10.50^{\text {cde }}$ & $9.70^{\mathrm{b}}$ & $8.98^{\mathrm{b}}$ & 9.79 \\
\hline Goldmine/Warrior/Butte & $40 / 20 / 40$ & $9.35^{\text {cde }}$ & $10.47^{\mathrm{b}}$ & $7.03^{\text {bcd }}$ & 8.95 \\
\hline Warrior/Scout & $50 / 50$ & $10.57^{\text {cde }}$ & $9.76^{\mathrm{b}}$ & $9.39^{\mathrm{b}}$ & 9.87 \\
\hline Scout/Shawnee/Butte & $40 / 20 / 40$ & $8.50^{\mathrm{de}}$ & $10.27^{\mathrm{b}}$ & $7.27^{\mathrm{bcd}}$ & 8.68 \\
\hline Warrior/Shawnee/Butte & $40 / 20 / 40$ & $11.00^{\mathrm{cd}}$ & $9.53^{\mathrm{b}}$ & $8.70^{\mathrm{b}}$ & 9.74 \\
\hline
\end{tabular}

${ }^{\dagger}$ Different letters represent significant differences in Bonferroni test, $\alpha=0.05 \%$

$\S$ The number followed by abbreviation indicates different ratio of each species in the seeding mixture

[12]. In this present study, each warm-season grass responded differently to precipitation early in the growing season (cumulative precipitation from April-July) at Urbana and Mead (Fig. 1). The biomass productivity of $M . \times$ giganteus at Mead, NE had a high positive correlation with April and July precipitation. At Urbana, $M . \times$ giganteus's biomass production was still positively correlated, although not significantly. This is in agreement with Heaton et al. [8] and Richter et al. [32] in showing that growing season (AprilSeptember) precipitation a critical factor for perennial grasses to produce their full biomass yield potential. Shiflet and Dietz [38] also indicated that May-July precipitation can be used as an indicator to estimate big bluestem production and Hong et al. [9] found high correlation between 'Bison' big bluestem biomass yield and April-September precipitation in the northern Great Plains. In this current study, biomass yield of big bluestem was positively correlated with growing season precipitation at Urbana. However, both cultivars produced consistent yield at Urbana and Mead as long as precipitation from April-July was above $250 \mathrm{~mm}$. For switchgrass and indiangrass, however, biomass yield was not correlated with precipitation during the growing season at either location. Results indicated that switchgrass and indiangrass biomass yields did not significantly respond to seasonal precipitation as long as April-July precipitation was more than $200 \mathrm{~mm}$ and $300 \mathrm{~mm}$, respectively, for each species. Previous work by Lee and Boe [14], however reported the importance of April-May precipitation for switchgrass biomass yield when the precipitation was below the 30 -year average $(125 \mathrm{~mm})$ in central South Dakota. In the current study, 2012 was the only year 
Table 6 Biomass moisture content of warm-season grass cultivars grown in monoculture from 2010 to 2015 at Urbana, IL and Mead, NE and from 2010 to 2011 at Ames, IA. Moisture content was measured at harvest after a killing frost each year

Moisture content $\left(\mathrm{g} \mathrm{kg}^{-1}\right)$

Species $\quad 2010 \quad 2011 \quad 2012 \quad 2013 \quad 2014 \quad 2015 \quad$ Mean

(a) Urbana, IL

$\begin{array}{llllllll}\text { M. } \times \text { giganteus } & 29.4 & 39.5 & 33.0 & 33.6 & 34.1 & 21.9 & 31.9 \\ \text { Cave-in-Rock } & 28.8 & 31.0 & 22.7 & 26.7 & 18.0 & 17.9 & 24.2 \\ \text { Kanlow N1 } & 26.0 & 28.2 & 23.2 & 23.7 & 17.8 & 16.5 & 22.6 \\ \text { NE2K } & 22.5 & 28.6 & 18.9 & 22.4 & 18.3 & 16.9 & 21.3 \\ \text { Shawnee } & 27.4 & 32.2 & 22.7 & 26.3 & 19.0 & 19.0 & 24.4 \\ \text { Bonanza } & 11.8 & 40.2 & 15.4 & 29.8 & 17.7 & 35.6 & 25.1 \\ \text { Goldmine } & 13.4 & 35.2 & 14.4 & 30.4 & 19.9 & 38.2 & 25.3 \\ \text { Chief } & 12.5 & 24.3 & 15.0 & 16.1 & 16.6 & 17.8 & 17.1 \\ \text { Scout } & 13.0 & 23.8 & 16.2 & 17.2 & 18.0 & 21.2 & 18.2 \\ \text { Warrior } & 16.4 & 25.5 & 16.7 & 20.5 & 18.1 & 16.9 & 19.0 \\ \text { Mean } & 20.4 & 30.8 & 19.8 & 24.7 & 19.9 & 22.2 & \\ \text { LSD }(\alpha=0.05) & 2.04 & 5.59 & 2.28 & 6.17 & 3.22 & 4.47 & \\ \text { b) Mead, NE } & & & & & & & \\ \text { M. } \times \text { giganteus } & 53.0 & 42.9 & 31.9 & 36.9 & 37.5 & 55.4 & 42.9 \\ \text { Cave-in-Rock } & 47.0 & 39.1 & 25.8 & 34.1 & 38.9 & 49.6 & 39.1 \\ \text { Kanlow N1 } & 49.4 & 4.06 & 24.3 & 33.6 & 36.6 & 59.2 & 34.5 \\ \text { NE2K } & 35.6 & 31.2 & 24.8 & 24.8 & 30.7 & 40.2 & 31.2 \\ \text { Shawnee } & 45.8 & 36.1 & 22.7 & 31.6 & 34.6 & 45.8 & 36.1 \\ \text { Bonanza } & 40.9 & 24.6 & 17.4 & 18.5 & 23.9 & 33.4 & 26.5 \\ \text { Goldmine } & 45.1 & 31.5 & 20.3 & 23.1 & 29.6 & 39.2 & 31.5 \\ \text { Chief } & 27.9 & 24.1 & 23.6 & 18.2 & 24.0 & 26.8 & 24.1 \\ \text { Scout } & 33.4 & 26.6 & 22.6 & 16.6 & 26.3 & 34.1 & 26.6 \\ \text { Warrior } & 36.4 & 28.3 & 23.4 & 23.4 & 25.0 & 33.4 & 28.3 \\ \text { Mean } & 41.4 & 32.5 & 23.7 & 26.1 & 30.7 & 41.7 & \\ \text { LSD ( } \alpha=0.05) & 6.46 & 3.48 & 4.80 & 6.24 & 5.85 & 12.0 & \end{array}$

(c) Ames, IA

\begin{tabular}{llll} 
M. $\times$ giganteus & 17.0 & 11.9 & 14.5 \\
Cave-in-Rock & 25.2 & 19.5 & 22.4 \\
Kanlow N1 & 11.8 & 12.7 & 12.3 \\
NE2K & 15.1 & 11.6 & 13.4 \\
Shawnee & 11.8 & 11.8 & 11.8 \\
Bonanza & 16.1 & 21.1 & 18.6 \\
Goldmine & 23.4 & 16.2 & 19.8 \\
Chief & 22.5 & 21.5 & 22.0 \\
Scout & 11.0 & 12.7 & 11.9 \\
Warrior & 12.0 & 11.9 & 12.0 \\
Mean & 16.6 & 15.1 & \\
LSD $(\alpha=0.05)$ & 6.65 & 5.99 & \\
\hline
\end{tabular}

in which Urbana and Mead had a severe drought that resulted in a significant reduction in biomass yield across feedstocks, with the exception of switchgrass (Suppl. Table 1). Biomass yields of indiangrass and big bluestem in Urbana and Mead were $35-47 \%$ and $11-39 \%$ below average in 2012 when April-July precipitation was $50 \%$ and $70 \%$ below the 30 year average, respectively. In the two- and three-way mixtures of indiangrass, big bluestem, and switchgrass, no clear response to precipitation was observed. This may be because individual species within a mixture may respond differentially to water received during different times throughout the growing season. As a result, there was no relationship between total biomass yield and precipitation in mixed species stands as was also seen in a study by Wang et al. [52].

\section{Biomass Production and Species Diversity}

According to Picasso et al. [30], the relationship between biomass production and species diversity might be affected by the presence or absence of "driver" species, which are defined as certain species from which most of the biomass yield is achieved, either in monocultures or in mixtures. Hong et al. [9] reported that switchgrass yielded more than indiangrass and big bluestem in the northern Great Plains, and the biomass yield of switchgrass in monoculture and the two-way or threeway mixture of indiangrass and big bluestem with switchgrass were higher than the mixture that did not include switchgrass. This was also found in the current study. 'Bonanza' big bluestem mixtures containing 'Shawnee' switchgrass resulted in yields that were closer to the 'Shawnee' monoculture rather than either 'Bonanza' or 'Scout' indiangrass monocultures at Urbana, while a binary mixture of 'Bonanza' and 'Scout' produced a yield that was not significantly different from 'Scout' indiangrass (Table 4). However, there was no consistent difference in biomass yields between big bluestem monocultures and two- or three-way mixtures at Urbana and Mead. Although a three-way mixture containing side oats grama produced significantly lower biomass than other mixtures at Urbana (Table 4).

Compatibility in two- and three-way mixtures, which indicates the ability of different species to survive and yield when planted together, was different depending on grass species (Suppl. Table 2). Although indiangrass is one of the dominant warm-season perennial grasses in the tallgrass prairie [18, 53], studies found the ratio of indiangrass declined in mixtures containing big bluestem or switchgrass [9, 40]. In eastern Nebraska tallgrass prairies, big bluestem was found to comprise $42-62 \%$ of the total herbaceous standing crop, while indiangrass comprised only $14-16 \%$ of the total herbaceous standing crop [20]. Mulkey et al. [26] reported that switchgrass was compatible with big bluestem in South Dakota and big bluestem was likely to be more competitive than switchgrass, while switchgrass out-performed big bluestem in a big bluestem-switchgrass mixture in Oklahoma [40]. In addition, three-way mixtures, which were composed of big bluestem, indiangrass, and sideoats grama, produced lower biomass yields than other mixtures at Urbana, while big bluestem, 

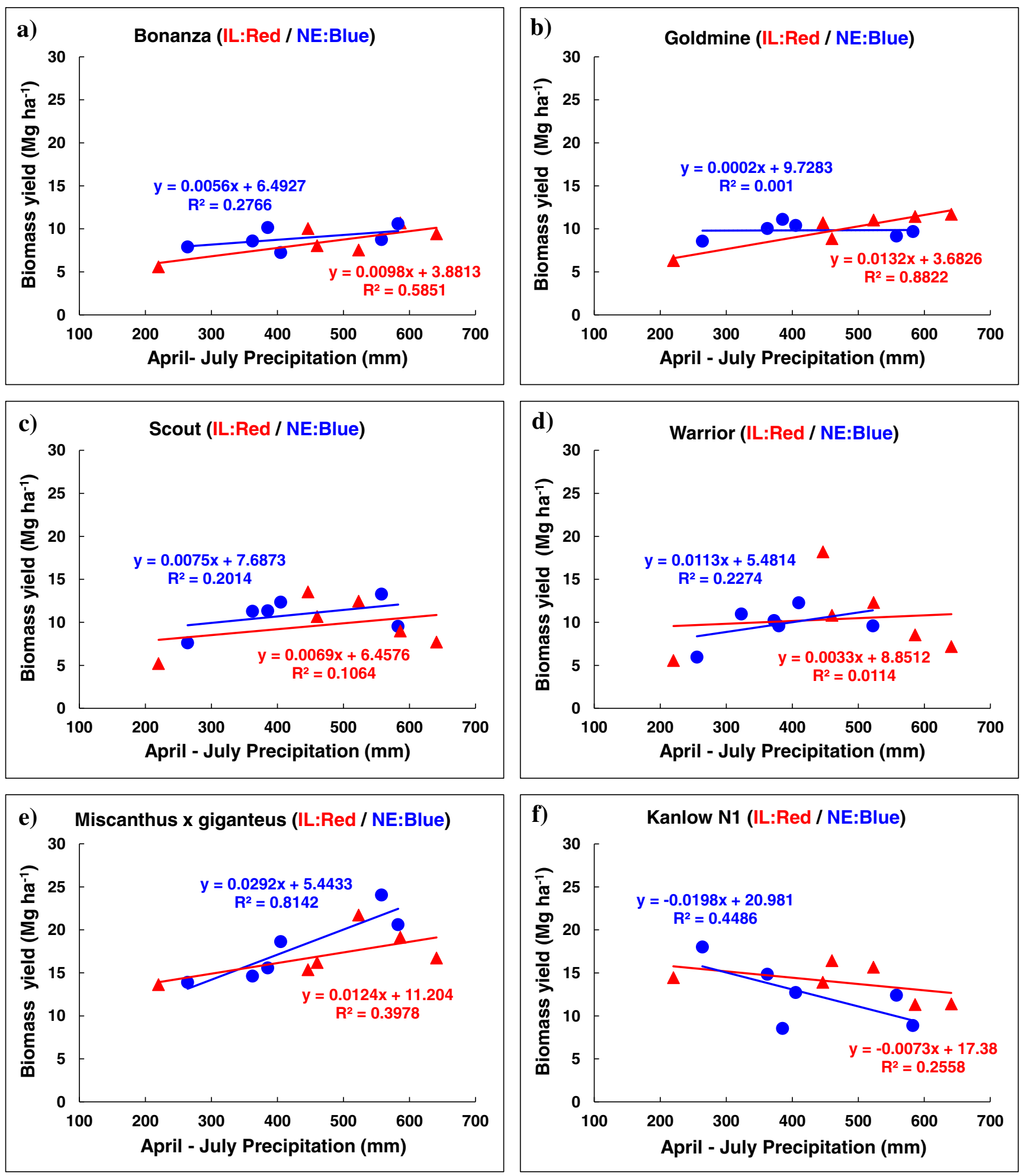

Fig. 1 Relationships between annual biomass dry matter yield and April and July precipitation from 2010 through 2015 at Urbana, IL and Mead, NE. (A) Big bluestem: (a) Bonanza and (b) Goldmine. (B) Indiangrass:

(a) Scout and (b) Warrior. (C) Miscanthus $\times$ giganteus. (D) Switchgrass: Kanlow N1

indiangrass, and sideoats grama produced yields comparable to other mixtures at Mead. This is due to the species composition change which was observed in the three-way mixture at

Urbana and Mead. As species compositions shifted after establishment with harvest management, big bluestem was a dominant species in two-way mixture plots and big bluestem 
and switchgrass were dominant species in three-way mixture plots.

Stand age and environmental conditions influenced the pattern of biomass production change of individual species over the 6-year study (Suppl. Table 1 and Suppl. Fig. 1). The biomass yields of $M . \times$ giganteus and big bluestem did not decrease at Urbana or Mead, while switchgrass and indiangrass yields tended to decrease as the stands aged (Suppl. Fig. 1). A yield decline in switchgrass was observed in 2013 and 2014 at Mead. The reason may have been due to the combination of a water deficit (due to drought conditions) and a late frost, which may have caused damage to Kanlow N1 switchgrass. July through August of 2012 was the second driest year recorded during a period from 1887 to 2014 in Mead, NE. A lack of precipitation resulted in lower soil moisture and subsequent soil moisture depletion in the root zone, which impacted the following year. Moreover, March of 2014 at Mead was the fifth driest month during 1887-2014 and frost damage occurred on May 15 and 17 in 2014.

\section{Conclusion}

The 6-year field experiment improves our understanding of the biomass production potential of native warm-season grasses in monocultures and mixtures in the Midwest. At all locations, $M . \times$ giganteus and 'Kanlow N1' produced the highest biomass yields in monocultures, and mixtures containing switchgrass and big bluestem had the highest mixture yields. All warm-season species tested in either monoculture or mixture conditions in this study consistently produced biomass as long as April-July precipitations were near or above the average precipitation $(300 \mathrm{~mm})$ of the regions. The 6-year field study results suggest mixtures provided no yield advantage over monocultures for bioenergy feedstocks in Illinois and Nebraska. However, compatibility and seasonal precipitation should be considered when developing warm-season grass mixtures to produce consistent biomass yields, confer resilience to environmental fluctuation, and improve biodiversity.

\section{References}

1. Boe A, Springer T, Lee DK, Rayburn AL, Gonzalez-Hernandez J (2013) Underutilized grasses. In: Malay CS et al. (eds) Bioenergy feedstocks: breeding and genetics. Wiley, New York, pp173-205

2. Bouton JH (2010) Future developments and uses. In: Boller B et al. (eds), Fodder Crops and Amenity Grasses. Springer, New York, pp. 201-209

3. Coblentz WK, Turner JE, Scarbrough DA, Lesmeister KE, Johnson ZB, Kellogg DW, Coffey KP, McBeth LJ, Weyers JS (2000) Storage characteristics and nutritive value changes in bermudagrass hay as affected by moisture content and density of rectangular bales. Crop Sci 40:1375-1383

4. Danalatos NG, Archontoulis SV, Mitsios I (2007) Potential growth and biomass productivity of Miscanthus $\times$ giganteus as affected by plant density and $\mathrm{N}$-fertilization in Central Greece. Biomass Bioenergy 31:145-152

5. Elmqvist T, Folke C, Nyström M, Peterson G, Bengtsson J, Walker B, Norberg J (2003) Response diversity, ecosystem change, and resilience. Front Ecol Environ 1:488-494

6. FAO (1997) Report of the study on CGIAR research priorities for marginal lands. Consultative Group on International Agricultural Research, Food and Agriculture Organization of the United Nations; FAO web. http://www.fao.org/Wairdocs/TAC/X5784E/ x5784e02.htm. Accessed 3 March 1997

7. Gonzalez-Hernandez JL, Sarath G, Stein JM, Owens V, Gedye K, Boe A (2009) A multiple species approach to biomass production from native herbaceous perennial feedstocks. In: In Vitro Cell Dev Biol Plant, vol 5, pp 267-281

8. Heaton EA, Long SP, Voigt TB, Jones MB, Clifton-Brown J (2004) Miscanthus for renewable energy generation: European Union experience and projections for Illinois. Mitig Adapt Strat G1 9:433451

9. Hong CO, Owens VN, Lee DK, Boe A (2012) Switchgrass, big bluestem, and indiangrass monocultures and their two-and threeway mixtures for bioenergy in the Northern Great Plains. Bioenergy Res 6:229-239

10. Energy Independence and Security Act of 2007 (EISA) (2007) Pub L 2008(110-140):121

11. Jones PG, Thornton PK (2003) The potential impacts of climate change on maize production in Africa and Latin America in 2055. Glob Environ Chang 13:51-59

12. Kardol P, Campany CE, Souza L, Norby RJ, Weltzin JF, Classen AT (2010) Climate change effects on plant biomass alter dominance patterns and community evenness in an experimental old-field ecosystem. Glob Chang Biol 16:2676-2687

13. Langholtz MH, Stokes BJ, Eaton LM (2016) 2016 Billion-ton report: advancing domestic resources for a thriving bioeconomy, volume 1: economic availability of feedstock. Oak Ridge National Laboratory, Oak Ridge, Tennessee, managed by UT-Battelle, LLC for the US Department of Energy.2016:1-411

14. Lee DK, Boe A (2005) Biomass production of switchgrass in central South Dakota. Crop Sci 45:2583-2590

15. Lee MS, Wycislo A, Guo J, Lee DK, Voigt T (2017) Nitrogen fertilization effects on biomass production and yield components of Miscanthus $\times$ giganteus. Front Plant Sci 8:544

16. Lin BB (2011) Resilience in agriculture through crop diversification: adaptive management for environmental change. BioScience 61:183-193

17. Martin AR, Moomaw RS, Vogel KP (1982) Warm-season grass establishment with atrazine. Agron J 74:916-920

18. McMillan C (1959) The role of ecotypic variation in the distribution of the central grassland of North America. Ecol Monogr 29:285-308

19. Mitchell CE, Tilman D, Groth JV (2002) Effects of grassland plant species diversity, abundance, and composition on foliar fungal disease. Ecology 83:1713-1726

20. Mitchell RB, Masters RA, Waller SS, Moore KJ, Young LJ (1996) Tallgrass prairie vegetation response to spring burning dates, fertilizer, and atrazine. J Range Manage 1:131-136

21. Mitchell R, Owens V, Gutterson N, Richard E, Barney J (2011) Herbaceous perennials: placement, benefits and incorporation challenges in diversified landscapes. ARS USDA Submissions

22. Mitchell R, Vogel KP, Sarath G (2008) Managing and enhancing switchgrass as a bioenergy feedstock. Biofuels Bioprod Biorefin 2: $530-539$ 
23. Mitchell RB, Vogel KP (2004) Indiangrass. p. 937-953. In: L.E. Moser et al. (eds.), Warm-season (C4) grasses. ASA/CSSA/SSSA Special Publ. 45, Madison, WI

24. Mitchell RB, Schmer MR, Anderson WF, Jin V, Balkcom KS, Kiniry J, Coffin A, White P (2016) Dedicated energy crops and crop residues for bioenergy feedstocks in the central and eastern USA. Bioenergy Res 9:384-398

25. Moser LE, Burson BL, Sollenberger LE (2004) Warm-season $\left(\mathrm{C}_{4}\right)$ grass overview. In: Moser LE et al.(eds) Warm-season (C4) Grass, ASA, CSSA, and SSSA, Madison, pp 1-14

26. Mulkey VR, Owens VN, Lee DK (2008) Management of warmseason grass mixtures for biomass production in South Dakota USA. Bioresour Technol 99:609-617

27. Parrish DJ, Fike JH (2009) Selecting, establishing, and managing switchgrass (Panicum virgatum) for biofuels. In: Jonathan RM (ed) Biofuels: methods and protocols. Humana Press Inc, New York, pp 27-40

28. Paruelo JM, Lauenroth WK, Burke IC, Sala OE (1999) Grassland precipitation-use efficiency varies across a resource gradient. Ecosystems 2:64-68

29. Perrin R, Vogel K, Schmer M, Mitchell R (2008) Farm-scale production cost of switchgrass for biomass. Bioenergy Res 1:91-97

30. Picasso VD, Brummer EC, Liebman M, Dixon PM, Wilsey BJ (2008) Crop species diversity affects productivity and weed suppression in perennial polycultures under two management strategies. Crop Sci 48:331-342

31. Pyter, R. E. Heaton. F. Dohleman, T. Voigt, S. Long. 2009. Agronomic experiences with Miscanthus $\mathrm{x}$ giganteus in Illinois, USA. Biofuels. Methods in molecular biology (methods and protocols), vol 581. Humana Press, Totowa, NJ

32. Richter GM, Riche AB, Dailey AG, Gezan SA, Powlson DS (2008) Is UK biofuel supply from Miscanthus water-limited? Soil Use Manage 24:235-245

33. Porter PA, Mitchell RB, Moore KJ (2015) Reducing hypoxia in the Gulf of Mexico: reimagining a more resilient agricultural landscape in the Mississippi River watershed. J Soil Water Conserv 70:63-68

34. Quinn LD, Straker KC, Guo J, Kim S, Thapa S, Kling G, Lee DK, Voigt TB (2015) Stress-tolerant feedstocks for sustainable bioenergy production on marginal land. Bioenergy Res 8:1081-1100

35. Russelle MP, Morey RV, Baker JM, Porter PM, and Jung HG (2007) Comment on "Carbon-negative biofuels from low-input high-diversity grassland biomass”. Science 316:1567

36. Sanderson MA, Adler PR, Boateng AA, Casler MD, Sarath G (2006) Switchgrass as a biofuels feedstock in the USA. Can J Plant Sci 86:1315-1325

37. Schmer MR, Vogel KP, Mitchell RB, Perrin RK (2008) Net energy of cellulosic ethanol from switchgrass. Proc Natl Acad Sci U S A 105:464-469
38. Shiflet TN, Dietz HE (1974) Relationship between precipitation and annual rangeland herbage production in southeastern Kansas. J Range Manag 1:272-276

39. Sosa A, Acuna M, McDonnell K, Devlin G (2015) Managing the moisture content of wood biomass for the optimisation of Ireland's transport supply strategy to bioenergy markets and competing industries. Energy 86:354-368

40. Springer TL, Aiken GE, McNew RW (2001) Combining ability of binary mixtures of native, warm-season grasses and legumes. Crop Sci 41:818-823

41. Tahir MH, Casler MD, Moore KJ, Brummer EC (2011) Biomass yield and quality of reed canarygrass under five harvest management systems for bioenergy production. BioEnergy Res 4:111-119

42. Tengö M, Belfrage K (2004) Local management practices for dealing with change and uncertainty: a cross-scale comparison of cases in Sweden and Tanzania. Ecol Soc 9:4

43. Tilman D, Hill J, Lehman C (2006) Carbon-negative biofuels from low-input high-diversity grassland biomass. Science 314:1598-1600

44. Vogel KP, Hopkins AA, Moore KJ, Johnson KD, Carlson IT (1996) Registration of 'Shawnee' switchgrass. Crop Sci 36:1713

45. Vogel KP, Masters RA (2001) Frequency grid: a simple tool for measuring grassland establishment. J Range Manag 1:653-655

46. Vogel KP, Mitchell RB, Casler M, Sarath G (2014a) Registration of 'liberty' switchgrass. J Plant Regist 8:242-247

47. Vogel KP, Mitchell RB, Klopfenstein TJ, Anderson BE (2006a) Registration of 'Bonanza' big bluestem. Crop Sci 46:2313-2314

48. Vogel KP, Mitchell RB, Klopfenstein TJ, Anderson BE (2006b) Registration of 'Goldmine' big bluestem. Crop Sci 46:2314-2315

49. Vogel KP, Mitchell RB, Gorz HJ, Haskins FA, Newell LC, Klopfenstein TJ, Erickson G, Anderson BE (2010) Registration of 'Warrior', 'Scout', and 'Chief' Indiangrass. J Plant Regist 4: $115-122$

50. Vogel KP, Sarah G, Saathoff AJ, Mitchell RB (2011) Switchgrass. In: Halford NG, Karp A (eds) Energy crop. Royal Society of Chemistry, Cambridge, UK, pp 314-380

51. Vogel KP, Mitchell RB, Casler MD, Sarath G (2014b) Registration of 'Liberty' switchgrass. J Plant Registrations 8:242-247

52. Wang DA, Lebauer DS, Dietze MC (2010) A quantitative review comparing the yield of switchgrass in monocultures and mixtures in relation to climate and management factors. GCB Bioenergy 2: $16-25$

53. Weaver JE, Fitzpatrick TJ (1935) The prairie. Ecol Monogr 4:109_ 295

54. Yachi S, Loreau M (1999) Biodiversity and ecosystem productivity in a fluctuating environment: the insurance hypothesis. Proc Natl Acad Sci U S A 96:1463-1468

55. Zhu Y, Chen H, Fan J, Wang Y (2000) Genetic diversity and disease control in rice. Nature 406:718-722 
Supplementary Table 1. Biomass dry matter yields and coefficient of variance in monoculture and mixture treatment from 2010 to 2015 at Urbana, IL, Mead, NE, and Ames, IA.

a) Urbana, IL

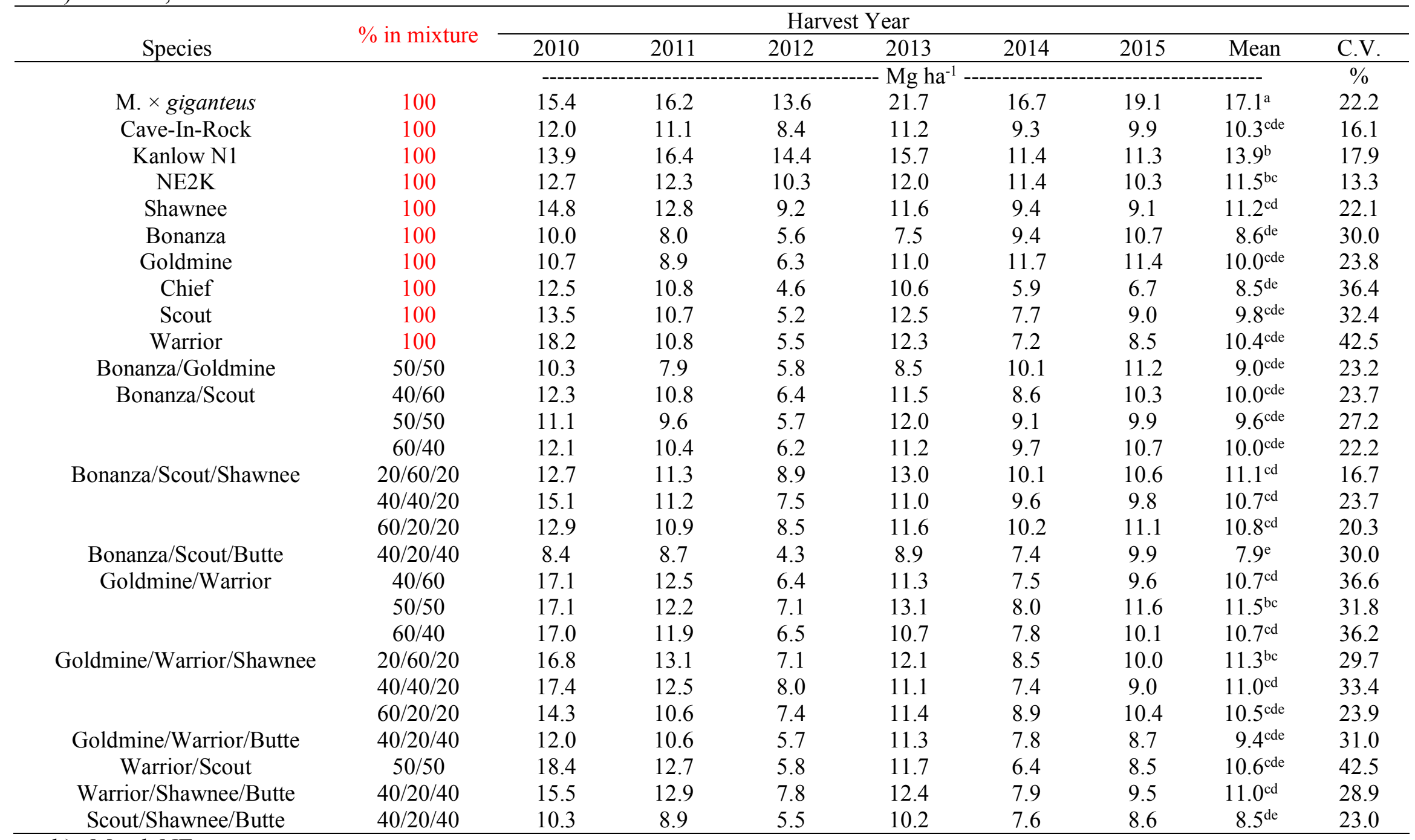

b) Mead, NE 


\begin{tabular}{|c|c|c|c|c|c|c|c|c|c|}
\hline Species & & 2010 & 2011 & 2012 & 2013 & 2014 & 2015 & Mean & C.V. \\
\hline \multicolumn{9}{|c|}{ 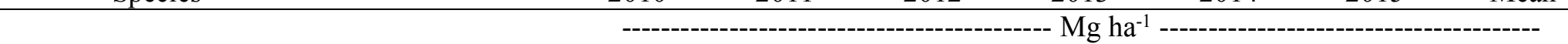 } & $\%$ \\
\hline M. $\times$ giganteus & 100 & 24.0 & 14.6 & 13.9 & 18.6 & 15.6 & 20.6 & $17.9^{\mathrm{a}}$ & 35.9 \\
\hline Cave-In-Rock & 100 & 9.8 & 9.7 & 11.1 & 9.8 & 7.8 & 9.4 & $9.6^{\mathrm{b}}$ & 18.9 \\
\hline Kanlow N1 & 100 & 12.4 & 14.8 & 18.0 & 12.7 & 8.5 & 8.9 & $12.6^{\mathrm{b}}$ & 31.3 \\
\hline NE2K & 100 & 9.4 & 9.4 & 10.6 & 10.5 & 9.5 & 10.2 & $9.9^{b}$ & 10.7 \\
\hline Shawnee & 100 & 10.6 & 10.3 & 12.5 & 10.8 & 9.0 & 9.9 & $10.5^{b}$ & 14.7 \\
\hline Bonanza & 100 & 8.8 & 8.6 & 7.9 & 7.2 & 10.2 & 10.6 & $8.9^{\mathrm{b}}$ & 24.9 \\
\hline Goldmine & 100 & 9.2 & 10.0 & 8.6 & 10.4 & 11.1 & 9.7 & $9.8^{\mathrm{b}}$ & 21.4 \\
\hline Chief & 100 & 12.4 & 8.7 & 5.7 & 9.0 & 10.1 & 9.0 & $9.1^{\mathrm{b}}$ & 31.2 \\
\hline Scout & 100 & 13.3 & 11.3 & 7.6 & 12.3 & 11.3 & 9.5 & $10.9^{b}$ & 24.9 \\
\hline Warrior & 100 & 12.3 & 11.0 & 6.0 & 9.6 & 10.2 & 9.6 & $9.8^{b}$ & 27.3 \\
\hline Bonanza/Goldmine & $50 / 50$ & 8.5 & 10.6 & 7.9 & 7.9 & 10.1 & 9.2 & $9.0^{\mathrm{b}}$ & 22.1 \\
\hline \multirow[t]{3}{*}{ Bonanza/Scout } & $40 / 60$ & 9.4 & 9.7 & 7.6 & 10.0 & 10.8 & 9.3 & $9.5^{\mathrm{b}}$ & 18.8 \\
\hline & $50 / 50$ & 9.9 & 9.4 & 9.1 & 10.4 & 10.5 & 8.3 & $9.6^{\mathrm{b}}$ & 17.4 \\
\hline & $60 / 40$ & 9.2 & 10.0 & 8.2 & 9.6 & 9.8 & 9.8 & $9.4^{\mathrm{b}}$ & 14.5 \\
\hline \multirow[t]{3}{*}{ Bonanza/Scout/Shawnee } & $20 / 60 / 20$ & 11.4 & 10.8 & 10.9 & 9.4 & 8.5 & 8.8 & $10.0^{\mathrm{b}}$ & 18.0 \\
\hline & $40 / 40 / 20$ & 10.3 & 10.9 & 12.1 & 11.4 & 9.4 & 9.2 & $10.5^{b}$ & 17.4 \\
\hline & $60 / 20 / 20$ & 10.4 & 10.7 & 11.6 & 10.1 & 9.2 & 9.5 & $10.2^{b}$ & 16.4 \\
\hline Bonanza/Scout/Butte & $40 / 20 / 40$ & 9.2 & 9.1 & 7.3 & 8.0 & 10.4 & 11.1 & $9.2^{\mathrm{b}}$ & 28.3 \\
\hline \multirow[t]{3}{*}{ Goldmine/Warrior } & $40 / 60$ & 11.7 & 10.4 & 8.4 & 9.8 & 10.5 & 11.0 & $10.2^{\mathrm{b}}$ & 16.9 \\
\hline & $50 / 50$ & 10.3 & 11.6 & 7.0 & 10.1 & 10.7 & 9.3 & $9.8^{b}$ & 22.0 \\
\hline & $60 / 40$ & 11.7 & 9.8 & 8.0 & 10.6 & 10.0 & 9.0 & $9.9^{\mathrm{b}}$ & 20.9 \\
\hline \multirow[t]{3}{*}{ Goldmine/Warrior/Shawnee } & $20 / 60 / 20$ & 12.9 & 10.8 & 12.5 & 10.7 & 9.2 & 9.1 & $10.9^{b}$ & 21.7 \\
\hline & $40 / 40 / 20$ & 12.7 & 10.6 & 11.3 & 8.4 & 8.4 & 8.5 & $10.0^{\mathrm{b}}$ & 26.2 \\
\hline & $60 / 20 / 20$ & 10.9 & 9.5 & 10.7 & 8.9 & 8.6 & 9.6 & $9.7^{\mathrm{b}}$ & 18.3 \\
\hline Goldmine/Warrior/Butte & $40 / 20 / 40$ & 10.1 & 10.1 & 9.4 & 10.3 & 11.8 & 11.0 & $10.5^{\mathrm{b}}$ & 22.3 \\
\hline Warrior/Scout & $50 / 50$ & 11.0 & 10.8 & 6.6 & 9.1 & 9.5 & 11.5 & $9.8^{\mathrm{b}}$ & 29.0 \\
\hline Warrior/Shawnee/Butte & $40 / 20 / 40$ & 11.1 & 9.2 & 9.6 & 10.2 & 7.7 & 9.5 & $9.5^{\mathrm{b}}$ & 18.4 \\
\hline Scout/Shawnee/Butte & $40 / 20 / 40$ & 12.4 & 10.7 & 10.0 & 9.9 & 8.8 & 9.9 & $10.3^{b}$ & 17.6 \\
\hline
\end{tabular}

c) Ames, IA

\begin{tabular}{|c|c|c|c|c|c|}
\hline \multirow[b]{2}{*}{ Species } & \multirow{2}{*}{$\%$ in mixture } & \multicolumn{3}{|c|}{ Harvest Year } & \\
\hline & & 2010 & 2011 & Mean & C.V. \\
\hline
\end{tabular}




\begin{tabular}{|c|c|c|c|c|c|}
\hline \multicolumn{2}{|l|}{ M. $\times$ giganteus } & 13.9 & 13.0 & $13.4^{\mathrm{a}}$ & 39.2 \\
\hline \multicolumn{2}{|l|}{ Cave-In-Rock } & 6.1 & 8.1 & $7.1^{\mathrm{bcd}}$ & 22.0 \\
\hline \multicolumn{2}{|l|}{ Kanlow N1 } & 8.4 & 9.8 & $9.1^{\mathrm{b}}$ & 19.2 \\
\hline \multicolumn{2}{|l|}{ NE2K } & 7.0 & 7.0 & $7.0^{\mathrm{bcd}}$ & 21.3 \\
\hline \multicolumn{2}{|l|}{ Shawnee } & 7.3 & 7.6 & $7.4^{\mathrm{bcd}}$ & 18.2 \\
\hline \multicolumn{2}{|l|}{ Bonanza } & 5.4 & 4.6 & $5.0^{\mathrm{cd}}$ & 24.4 \\
\hline \multicolumn{2}{|l|}{ Goldmine } & 6.7 & 6.1 & $6.4^{\mathrm{bcd}}$ & 13.9 \\
\hline \multicolumn{2}{|l|}{ Chief } & 7.4 & 6.9 & $7.2^{\mathrm{bcd}}$ & 19.1 \\
\hline \multicolumn{2}{|l|}{ Scout } & 7.8 & 9.2 & $8.5^{\mathrm{b}}$ & 18.2 \\
\hline \multicolumn{2}{|l|}{ Warrior } & 9.3 & 8.1 & $8.7^{\mathrm{b}}$ & 14.7 \\
\hline Bonanza/Goldmine & $50 / 50$ & 4.7 & 4.5 & $4.6^{\mathrm{bcd}}$ & 19.4 \\
\hline \multirow[t]{3}{*}{ Bonanza/Scout } & $40 / 60$ & 6.1 & 7.8 & $7.0^{\mathrm{bcd}}$ & 16.8 \\
\hline & $50 / 50$ & 5.4 & 7.7 & $6.5^{\mathrm{bcd}}$ & 21.6 \\
\hline & $60 / 40$ & 6.8 & 6.6 & $6.7^{\mathrm{bcd}}$ & 13.5 \\
\hline \multirow[t]{3}{*}{ Bonanza/Scout/Shawnee } & $20 / 60 / 20$ & 6.7 & 8.6 & $7.6^{\mathrm{bcd}}$ & 23.9 \\
\hline & $40 / 40 / 20$ & 7.8 & 8.4 & $8.1^{\mathrm{bc}}$ & 13.3 \\
\hline & $60 / 20 / 20$ & 6.7 & 7.1 & $6.9^{\text {bcd }}$ & 12.1 \\
\hline Bonanza/Scout/Butte & $40 / 20 / 40$ & 5.9 & 5.6 & $5.8^{\mathrm{bcd}}$ & 28.8 \\
\hline \multirow{3}{*}{ Goldmine/Warrior } & $40 / 60$ & 9.4 & 9.0 & $9.2^{\mathrm{b}}$ & 14.4 \\
\hline & $50 / 50$ & 8.2 & 8.3 & $8.7^{\mathrm{b}}$ & 17.7 \\
\hline & $60 / 40$ & 7.3 & 8.7 & $8.0^{\mathrm{bcd}}$ & 21.3 \\
\hline \multirow{3}{*}{ Goldmine/Warrior/Shawnee } & $20 / 60 / 20$ & 9.2 & 9.2 & $9.2^{\mathrm{b}}$ & 9.6 \\
\hline & $40 / 40 / 20$ & 6.4 & 9.1 & $7.7^{\mathrm{bcd}}$ & 27.8 \\
\hline & $60 / 20 / 20$ & 8.3 & 9.6 & $9.0^{\mathrm{b}}$ & 14.6 \\
\hline \multirow{2}{*}{$\begin{array}{c}\text { Goldmine/Warrior/Butte } \\
\text { Warrior/Scout }\end{array}$} & $40 / 20 / 40$ & 6.4 & 7.6 & $7.0^{\mathrm{bcd}}$ & 20.9 \\
\hline & $50 / 50$ & 9.0 & 9.7 & $9.4^{\mathrm{b}}$ & 11.9 \\
\hline Warrior/Shawnee/Butte & $40 / 20 / 40$ & 7.4 & 10.0 & $8.7^{\mathrm{b}}$ & 17.4 \\
\hline Scout/Shawnee/Butte & $40 / 20 / 40$ & 6.7 & 8.1 & $7.2^{\mathrm{bcd}}$ & 21.8 \\
\hline
\end{tabular}


Supplementary Table 2. The average species composition of each mixture in 2009 and 2016 at Urbana, IL and Mead, NE.

A) Urbana, IL

\begin{tabular}{|c|c|c|c|c|c|c|c|c|}
\hline \multirow{2}{*}{$\begin{array}{c}\text { Year } \\
\text { Species }\end{array}$} & \multicolumn{4}{|c|}{$2009^{\dagger}$} & \multicolumn{4}{|c|}{2016} \\
\hline & $\mathrm{BB}$ & IN & SW & SO & BB & IN & SW & SO \\
\hline & \multicolumn{8}{|c|}{--------------------------------------------------------- $\%$} \\
\hline Bonanza + Scout $(40 / 60)$ & 40 & 60 & 0 & 0 & 58 & 17 & 25 & 0 \\
\hline Bonanza + Scout $(50 / 50)$ & 50 & 50 & 0 & 0 & 82 & 13 & 5 & 0 \\
\hline Bonanza + Scout $(60 / 40)$ & 60 & 40 & 0 & 0 & 66 & 18 & 16 & 0 \\
\hline Goldmine + Warrior $(50 / 50)$ & 50 & 50 & 0 & 0 & 75 & 7 & 18 & 0 \\
\hline Goldmine + Warrior $(60 / 40)$ & 60 & 40 & 0 & 0 & 80 & 5 & 15 & 0 \\
\hline \multicolumn{9}{|l|}{ Three-way mixture ( $\%$ in mixture) } \\
\hline Bonanza + Scout + Shawnee $(20 / 60 / 20)$ & 20 & 60 & 20 & 0 & 32 & 18 & 50 & 0 \\
\hline Bonanza + Scout + Shawnee $(40 / 40 / 20)$ & 40 & 40 & 20 & 0 & 60 & 18 & 22 & 0 \\
\hline Bonanza + Scout + Butte $(40 / 20 / 40)$ & 40 & 20 & 0 & 40 & 78 & 10 & 12 & 0 \\
\hline Goldmine + Warrior + Butte $(40 / 20 / 40)$ & 40 & 20 & 0 & 40 & 76 & 10 & 14 & 0 \\
\hline Scout + Shawnee + Butte $(40 / 20 / 40)$ & 0 & 40 & 20 & 40 & 48 & 34 & 18 & 0 \\
\hline Warrior + Shawnee + Butte $(40 / 20 / 40)$ & 0 & 40 & 20 & 40 & 28 & 29 & 43 & 0 \\
\hline
\end{tabular}

${ }^{\dagger}$ Species compositions in 2009 indicate the ratio of each species in the seeding mixture.

$4 \quad$ Species compositions in 2016 indicate field observations before biomass harvest in fall 2016.

B) Mead, NE 


\begin{tabular}{|c|c|c|c|c|c|c|c|c|}
\hline \multirow{2}{*}{$\begin{array}{l}\text { Year } \\
\text { Species }\end{array}$} & \multicolumn{4}{|c|}{$2010^{\dagger}$} & \multicolumn{4}{|c|}{$2016^{*}$} \\
\hline & BB & IN & SW & $\mathrm{SO}$ & BB & $\mathrm{IN}$ & SW & $\mathrm{SO}$ \\
\hline & \multicolumn{8}{|c|}{ 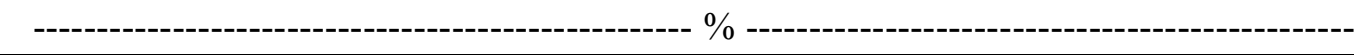 } \\
\hline \multicolumn{9}{|l|}{ Two-way mixture (\% in mixture) } \\
\hline Bonanza + Scout $(40 / 60)$ & 40 & 60 & 0 & 0 & 34 & 41 & 25 & 0 \\
\hline Bonanza + Scout $(50 / 50)$ & 50 & 50 & 0 & 0 & 50 & 50 & 0 & 0 \\
\hline Bonanza + Scout $(60 / 40)$ & 60 & 40 & 0 & 0 & 39 & 51 & 10 & 0 \\
\hline Goldmine + Warrior $(40 / 60)$ & 40 & 60 & 0 & 0 & 30 & 55 & 16 & 0 \\
\hline Goldmine + Warrior $(50 / 50)$ & 50 & 50 & 0 & 0 & 59 & 39 & 2 & 0 \\
\hline Goldmine + Warrior $(60 / 40)$ & 60 & 40 & 0 & 0 & 66 & 21 & 13 & 0 \\
\hline \multicolumn{9}{|l|}{ Three-way mixture ( $\%$ in mixture) } \\
\hline Bonanza + Scout + Shawnee $(20 / 60 / 20)$ & 20 & 60 & 20 & 0 & 14 & 20 & 66 & 0 \\
\hline Bonanza + Scout + Shawnee $(40 / 40 / 20)$ & 40 & 40 & 20 & 0 & 13 & 5 & 82 & 0 \\
\hline Bonanza + Scout + Shawnee $(60 / 20 / 20)$ & 60 & 20 & 20 & 0 & 31 & 9 & 60 & 0 \\
\hline Goldmine + Warrior + Shawnee $(20 / 60 / 20)$ & 20 & 60 & 20 & 0 & 25 & 5 & 70 & 0 \\
\hline Goldmine + Warrior + Shawnee $(40 / 40 / 20)$ & 40 & 40 & 20 & 0 & 34 & 10 & 56 & 0 \\
\hline Goldmine + Warrior + Shawnee $(60 / 20 / 20)$ & 60 & 20 & 20 & 0 & 32 & 12 & 56 & 0 \\
\hline Bonanza + Scout + Butte $(40 / 20 / 40)$ & 40 & 20 & 0 & 40 & 38 & 27 & 35 & 0 \\
\hline Goldmine + Warrior + Butte $(40 / 20 / 40)$ & 40 & 20 & 0 & 40 & 46 & 45 & 10 & 0 \\
\hline Scout + Shawnee + Butte $(40 / 20 / 40)$ & 0 & 40 & 20 & 40 & 10 & 7 & 83 & 0 \\
\hline Warrior + Shawnee + Butte $(40 / 20 / 40)$ & 0 & 40 & 20 & 40 & 25 & 12 & 63 & 0 \\
\hline
\end{tabular}

8 Species compositions in 2010 indicate the ratio of each species in the seeding mixture.

9 Species compositions in 2016 indicate field observations before biomass harvest in fall 2016. 
11 Supplementary Figure 1. Biomass dry matter yield $\left(\mathrm{Mg} \mathrm{ha}^{-1}\right)$ of indiangrass and switchgrass 12 pooled across cultivars for the second through sixth growing seasons.

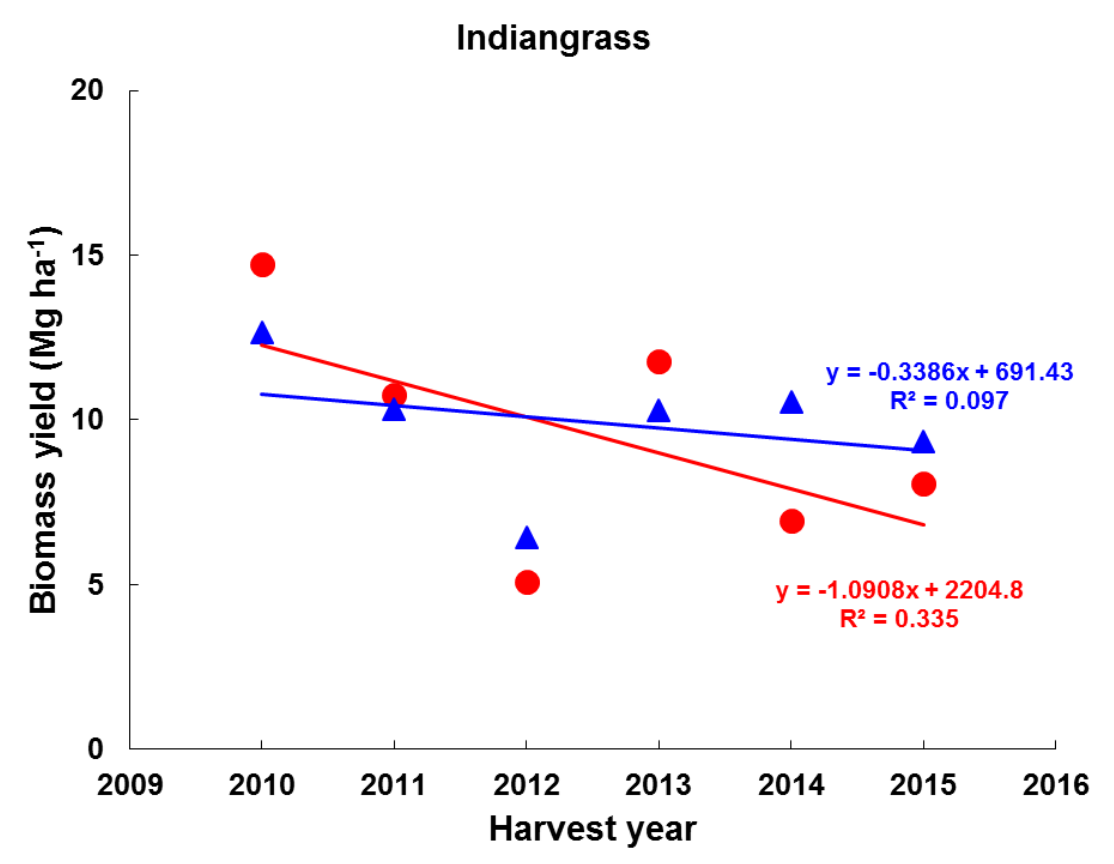

13

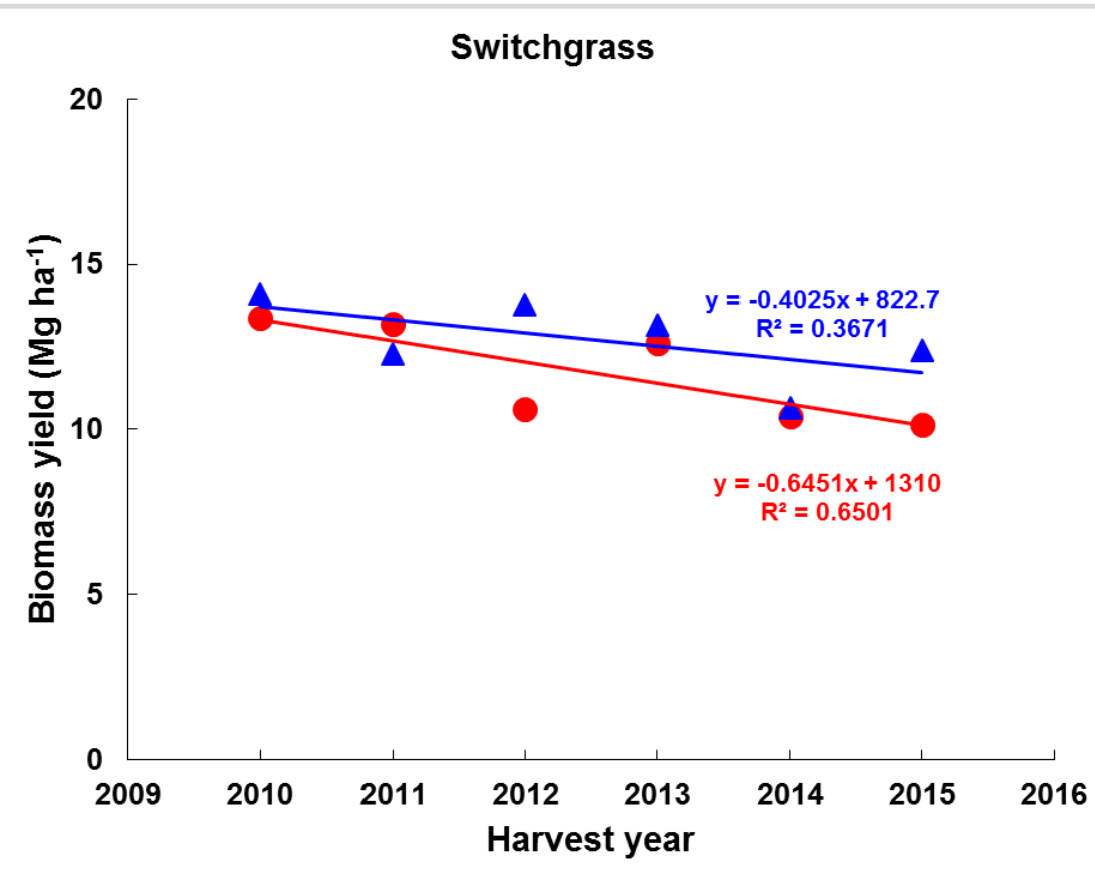

$14 \quad$ 'Red $(\bullet)$ and blue $(\boldsymbol{\Delta})$ represent Urbana and Mead, respectively.

15

16

17

18

19

20 
21 Supplementary Figure 2. Coefficient of variance for each treatment at each location by species 22 richness in monocultures and mixtures.
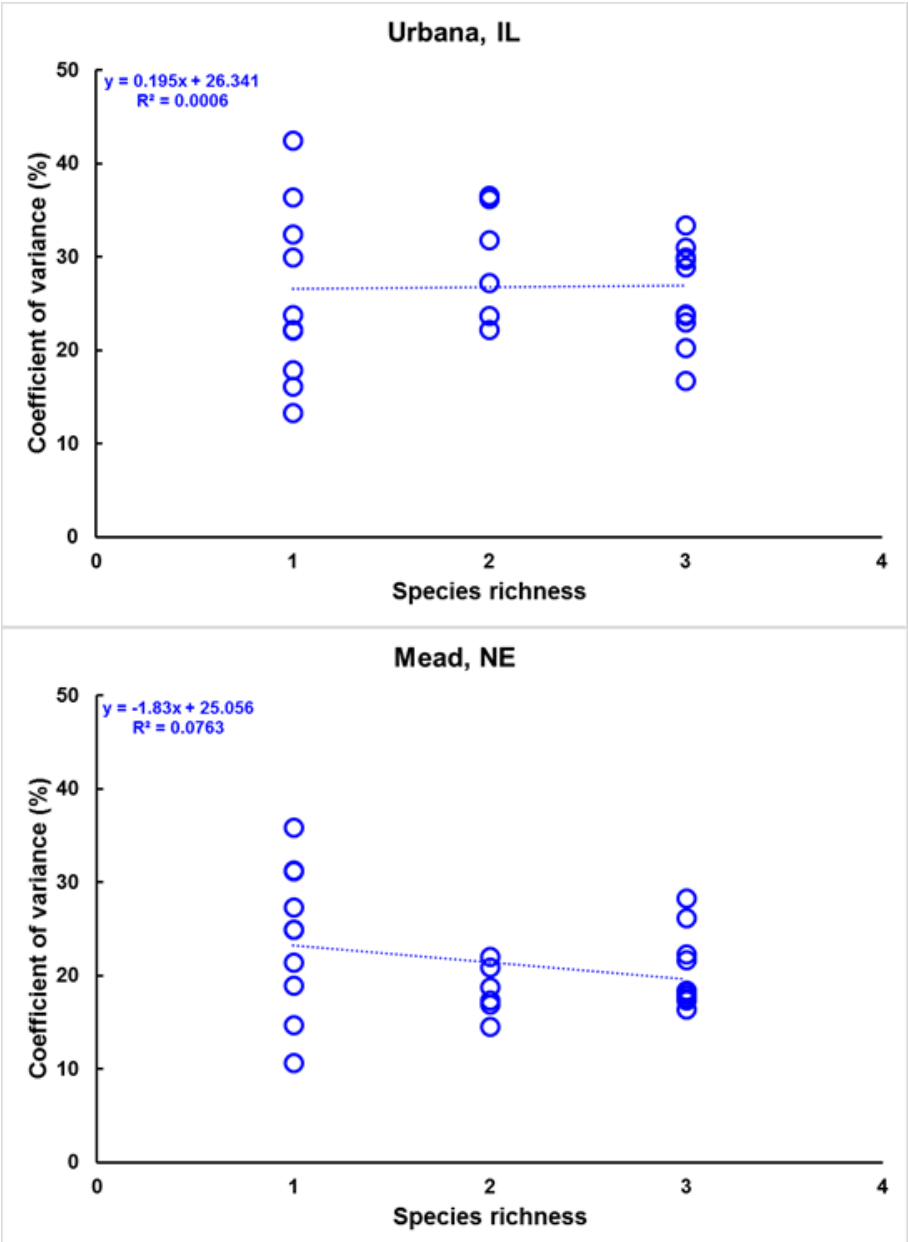

Ames, IA

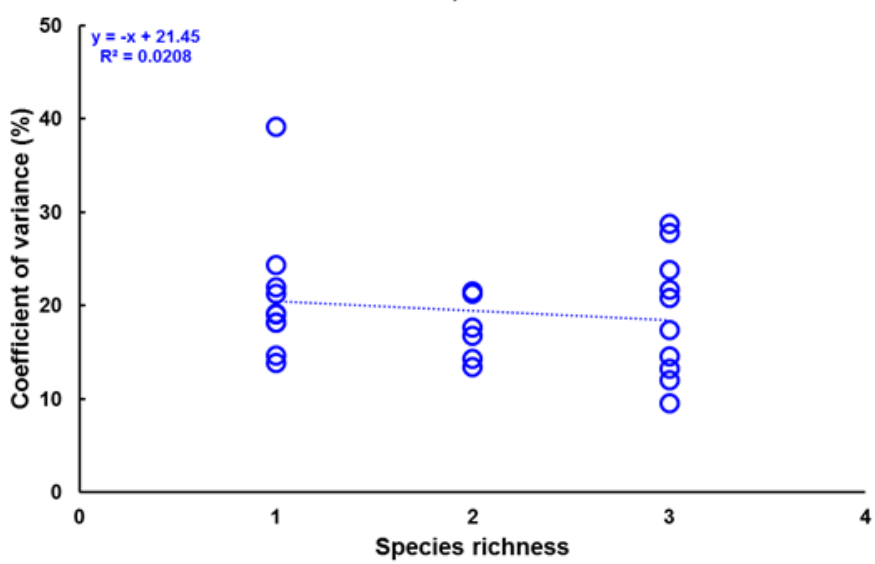

$24 \S$ Blends of 'Bonanza' and 'Goldmine' big bluestem and 'Warrior' and 'Scout' indiangrass were 25 not included in this analysis. 